\title{
INSTABILITY OF SOME IDEAL PLANE FLOWS*
}

\author{
ZHIWU LIN ${ }^{\dagger}$
}

\begin{abstract}
We prove the instability of large classes of steady states of the two-dimensional Euler equation. For an odd shear flow, beginning with the Rayleigh equation, we define a family of operators depending on some positive parameter. Then we use infinite determinants to keep track of the signs of the eigenvalues of these operators. The existence of purely growing modes follows from a continuation argument. Employing a new analysis of neutral modes together with a rigorous justification of Tollmien's classical method, we obtain a sharp condition for linear and hence nonlinear instability of a general class of bounded shear flows. We obtain similar results for bounded rotating flows and unbounded shear flows.
\end{abstract}

Key words. Rayleigh's equation, neutral limiting mode, shear flow instability

AMS subject classifications. 76E05, 76E09

DOI. $10.1137 /$ S0036141002406266

1. Introduction. In this paper, we study the hydrodynamic stability problem for plane shear flows and rotating flows. The purpose is to get some sufficient conditions for linear instability and hence nonlinear instability. For plane shear flows, this problem has a long history, going back to scientists such as Rayleigh and Kelvin in the nineteenth century. The vorticity form of the incompressible two-dimensional Euler equation in a bounded domain $D$ with smooth boundary $\partial D$ is

$$
\partial_{t} \omega+u \cdot \nabla \omega=0 \quad \text { in } \mathbf{R}_{t} \times D
$$

or

$$
\partial_{t} \Delta \psi+\frac{\partial \psi}{\partial y} \frac{\partial}{\partial x} \Delta \psi-\frac{\partial \psi}{\partial x} \frac{\partial}{\partial y} \Delta \psi=0
$$

where $\psi$ is the stream function, $\omega=-\Delta \psi$ is the vorticity, and $u=\left(\frac{\partial \psi}{\partial y},-\frac{\partial \psi}{\partial x}\right)$ is the velocity. We consider the basic steady state flow $\mathbf{U}_{0}=U(y) \mathbf{i}$, a parallel shear flow in the $x$-direction, in the flow domain $D=\left\{(x, y) \mid y_{1} \leq y \leq y_{2}\right\}$ with rigid walls at $y=y_{1}, y_{2}$. This means $u$ is tangential, or $\psi$ is constant on each wall. The linearized equation of (1) around $\mathbf{U}_{0}$ is

$$
\partial_{t} \Delta \tilde{\psi}+U \frac{\partial}{\partial x} \Delta \tilde{\psi}-U^{\prime \prime} \frac{\partial \tilde{\psi}}{\partial x}=0
$$

where $\tilde{\psi}$ is constant on $y=y_{j}(j=1,2)$. Taking $\tilde{\psi}=\phi(y) e^{i \alpha(x-c t)}$ with $\alpha$ the wave number (positive real) in the $x$-direction and $c=c_{r}+i c_{i}$ the complex wave speed, we obtain from (2) the Rayleigh equation

$$
(U-c)\left(\frac{d^{2}}{d y^{2}}-\alpha^{2}\right) \phi-U^{\prime \prime} \phi=0
$$

${ }^{*}$ Received by the editors April 24, 2002; accepted for publication (in revised form) January 31, 2003; published electronically July 18, 2003.

http://www.siam.org/journals/sima/35-2/40626.html

†Department of Mathematics, Brown University, Providence, RI 02912 (linzw@math.brown.edu). 
with $\phi\left(y_{1}\right)=\phi\left(y_{2}\right)=0$. We will also consider unbounded shear flows where one of $y_{1}, y_{2}$ is infinity, with the boundary condition $\phi(y) \rightarrow 0$ as $y \rightarrow \infty$.

So for shear flows, the instability problem is reduced to studying the Rayleigh equation (3). The flow is linearly unstable if some nontrivial solution to (3) with $c_{i}>0$ exists. A classical result of Lord Rayleigh [16] is the necessary condition for instability that the basic velocity profile should have an inflection point at some point $y=y_{s}$, that is, $U^{\prime \prime}\left(y_{s}\right)=0$. This condition was later improved by Fjørtoft [10]. Howard's semicircle theorem [14] says that any unstable eigenvalue $c=c_{r}+i c_{i}$ must lie in the semicircle

$$
\left(c_{r}-\frac{1}{2}\left(U_{\min }+U_{\max }\right)\right)^{2}+c_{i}^{2} \leq\left(\frac{1}{2}\left(U_{\min }-U_{\max }\right)\right)^{2} .
$$

However, very few sufficient conditions for instability are known. In 1935, Tollmien [23] obtained an unstable solution to (3) by formally perturbing around a neutral mode ( $c$ real) for symmetric flows in class $\mathcal{K}^{+}$(defined below). The original presentation was improved by C. C. Lin [17] and the asymptotic growth rate was found. Even in recent treatises such as [20], the main instability result mentioned is Tollmien's. However, as indicated by Friedlander and Howard [12], in all these references the existence of an unstable mode had to be assumed in a neighborhood of the neutral mode. The assumption of analytic dependence between the parameters $\alpha$ and $c$ (complex) also lacked justification. These assumptions are rigorously justified in this paper. Here we get a sharp condition for the instability of a class of flows.

Let us describe the setting of the problem. First we define a class of flows having some inflection point. By an inflection value we mean the value of $U$ at an inflection point.

DeFINITION 1.1. The flow $U(y)$ is in class $\mathcal{K}$ if $U$ is a $\mathbf{C}^{2}$ function on a interval $\left[y_{1}, y_{2}\right]$, and there exists some inflection value $U_{s}$ such that

$$
K(y):=-U^{\prime \prime}(y) /\left(U(y)-U_{s}\right)
$$

is nonnegative and bounded in $\left[y_{1}, y_{2}\right]$. If $K$ is positive on $\left[y_{1}, y_{2}\right]$, we say that $U$ is in class $\mathcal{K}^{+}$.

A typical example of such a flow is $U=\cos m y$ or $\sin m y$. Now we consider any flow in class $\mathcal{K}$. If $\left(\phi_{s}, \alpha_{s}\right)$ is a solution to the Sturm-Liouville problem

$$
\phi_{s}^{\prime \prime}-\alpha_{s}^{2} \phi_{s}+K \phi_{s}=0, \phi=0 \quad \text { at } y=y_{1}, y_{2},
$$

then $(\phi, \alpha, c)=\left(\phi_{s}, \alpha_{s}, U_{s}\right)$ is a special solution (a so-called neutral mode) to the Rayleigh equation (3). Let $\alpha_{\max }$ be the largest wave number so that a neutral mode exists. That is,

$$
-\alpha_{\max }^{2}=\inf _{\phi \in \mathbf{H}_{0}^{1}\left(y_{1}, y_{2}\right)} \frac{\int_{y_{1}}^{y_{2}}\left(\left|\phi^{\prime}\right|^{2}-K(y)|\phi|^{2}\right) d y}{\int_{y_{1}}^{y_{2}}|\phi|^{2} d y} .
$$

Throughout this paper, we assume that the right-hand side of (7) is negative. Otherwise, the shear flow was proved to be linearly stable by Drazin and Howard [8]. It was also proved in [8] that instability is possible only for wave numbers $\alpha$ such that $0<\alpha<\alpha_{\max }$. Howard [15] estimated the maximal number of possible unstable modes for a fixed wave number. However, it still was not clear whether there exists 
some unstable mode for each $\alpha$ in that range. Recently, Friedlander and Howard [12] studied the special flow $U(y)=\cos m y$, using a continued fractions technique and a numerical method. For this flow they proved that for all $0<\alpha<\alpha_{\max }$, there exists some growing mode for the Rayleigh equation.

In this paper, we rigorously prove that for any flow of class $\mathcal{K}^{+}$and for all $0<$ $\alpha<\alpha_{\max }$, there does indeed exist an unstable solution to the Rayleigh equation (3). This is our main theorem.

TheOrem 1.2. Suppose the steady state is in class $\mathcal{K}^{+}$. Let $-\alpha_{\max }^{2}$ be the lowest eigenvalue of $-\frac{d^{2}}{d y^{2}}-K(y)$, which is assumed to be negative. For all $\alpha \in\left(0, \alpha_{\max }\right)$, there is an unstable solution (with $\operatorname{Im} c>0$ ) to (3).

The unstable interval $\left(0, \alpha_{\max }\right)$ is sharp in the sense that there is linear stability if $\alpha \geq \alpha_{\max }$ or $-\frac{d^{2}}{d y^{2}}-K(y)$ is nonnegative. We can also treat plane rotating flows in an annulus. In this case, the analogue of the Rayleigh equation becomes

$$
(\Omega-c)\left(D_{*} D-n^{2} / r^{2}\right) \phi-r^{-1}\left(r D^{2} \Omega+3 D \Omega\right) \phi=0,
$$

with $\phi\left(R_{1}\right)=\phi\left(R_{2}\right)=0,0<R_{1} \leq r \leq R_{2}$. Here $\Omega(r)$ is the angular velocity of the steady state, $D_{*}=\frac{d}{d r}+\frac{1}{r}, D=\frac{d}{d r}$, and $n$ is some integer. We have the following result analogous to Theorem 1.2.

Theorem 1.3. For the rotating case, if

$$
K(r):=-\left(r D^{2} \Omega+3 D \Omega\right) /\left(\Omega-\Omega_{s}\right)
$$

is positive and $\Omega\left(R_{1}\right) \neq \Omega\left(R_{2}\right)$, then a necessary and sufficient condition for instability is that there exists $\alpha>1$ such that the equation

$$
\left(D_{*} D-\alpha^{2} / r^{2}\right) \phi+r^{-1} K(r) \phi=0
$$

has some nontrivial solution with $\phi\left(R_{1}\right)=\phi\left(R_{2}\right)=0$. This is equivalent to the condition

$$
-\alpha_{\max }^{2}:=\inf _{\phi \in \mathbf{H}_{0}^{1}\left(R_{1}, R_{2}\right)} \frac{\int_{R_{1}}^{R_{2}} r\left(\frac{d}{d r} \phi\right)^{2} d r-\int_{R_{1}}^{R_{2}} K(r) \phi^{2} d r}{\int_{R_{1}}^{R_{2}} \frac{1}{r} \phi^{2} d r}<-1 .
$$

In the case that $K(r)$ is positive and $\Omega\left(R_{1}\right)=\Omega\left(R_{2}\right)$, a sufficient condition for instability is that

$$
-\alpha_{\max }^{2}:=\inf _{\phi \in \mathbf{H}_{0}^{1}\left(R_{1}, R_{2}\right)} \frac{\int_{R_{1}}^{R_{2}} r\left(\frac{d}{d r} \phi\right)^{2} d r-\int_{R_{1}}^{R_{2}} K(r) \phi^{2} d r}{\int_{R_{1}}^{R_{2}} \frac{1}{r} \phi^{2} d r}<-4 .
$$

Let us return to shear flows that are not in class $\mathcal{K}^{+}$. If a shear flow is odd but there is no assumption on the sign of $K(y)$, we can still get a sufficient condition for instability.

Theorem 1.4. Assume $U(y)$ is odd in $[-a, a]$ and define

$$
K(y):=-U^{\prime \prime}(y) / U(y) .
$$

If $K$ is bounded and the operator $-\frac{d^{2}}{d y^{2}}-K(y)$ with zero boundary values at $\pm a$ has a negative eigenvalue, then there is a solution to the Rayleigh equation (3) with $c=i \lambda_{0}$ (here $\left.\lambda_{0}>0\right)$ for some range of wave numbers. Specifically, if $-\alpha_{0}^{2}<-\alpha_{1}^{2}<$ $\cdots<-\alpha_{k_{0}}^{2}<0$ denote all the negative eigenvalues of $-\frac{d^{2}}{d y^{2}}-K(y)$, then we have a 
purely growing instability for $\alpha$ belonging to the intervals $\left(\alpha_{1}, \alpha_{0}\right) \cup \cdots \cup\left(\alpha_{2 k-1}, \alpha_{2 k-2}\right)$ $\cdots \cup\left(\alpha_{k_{0}}, \alpha_{k_{0}-1}\right)\left(\right.$ if $k_{0}$ odd $)$ or to the intervals $\left(\alpha_{1}, \alpha_{0}\right) \cup \cdots \cup\left(\alpha_{2 k-1}, \alpha_{2 k-2}\right) \cdots$ $\cup\left(\alpha_{k_{0}-1}, \alpha_{k_{0}-2}\right)$ (if $k_{0}$ even $)$.

We can extend Theorems 1.2 and 1.4 to the case of unbounded shear flows.

TheOrem 1.5. (i) (class $\mathcal{K}^{+}$) Assume $U(y)$ is in $\mathbf{C}^{2}(-\infty,+\infty), U(y) \rightarrow$ $U( \pm \infty)$ as $y \rightarrow \pm \infty$, and $U(y)$ takes the values $U( \pm \infty)$ at only a finite number of points. We consider the flows such that $K(y)$ defined by $(5)$ is bounded, positive, and $\lim _{y \rightarrow \pm \infty} K(y)=0$. Let $-\alpha_{0}^{2}$ be the lowest eigenvalue of $-\frac{d^{2}}{d y^{2}}-K(y)$ on $\mathbf{H}^{2}(\mathbf{R})$, which is assumed to be negative. Then for each $\alpha$ in $\left(0, \alpha_{0}\right)$, there is instability. This condition is sharp in the sense that if $\alpha \geq \alpha_{0}$ or $-\frac{d^{2}}{d y^{2}}-K(y)$ is nonnegative, then there is linear stability. The same result holds for the shear flows defined in the half line.

(ii) (odd flows) Assume $U(y)$ is in $\mathbf{C}^{2}(-\infty,+\infty)$, odd, and $K(y)$ defined by (13) is bounded and $\lim _{y \rightarrow \pm \infty} K(y)=0$. If $-\alpha_{0}^{2}<-\alpha_{1}^{2}<\cdots<-\alpha_{k}^{2}<\cdots<0$ denote all the negative eigenvalues of the operator $-\frac{d^{2}}{d y^{2}}-K(y)$ on $\mathbf{H}^{2}(\mathbf{R})$, then we have a purely growing instability for $\alpha$ belonging to the intervals $\left(\alpha_{1}, \alpha_{0}\right) \cup\left(\alpha_{3}, \alpha_{2}\right) \cup \cdots \cup$ $\left(\alpha_{2 k-1}, \alpha_{2 k-2}\right) \cdots$.

Now let us sketch the main ideas of the proofs. For the proof of Theorem 1.4, we define a family of elliptic operators $A_{\lambda}$ depending on the positive parameter $\lambda$ where $c=i \lambda$. The problem is reduced to finding some $\lambda_{0}$ such that $A_{\lambda_{0}}$ has a kernel. The operator $A_{\lambda}$ is nonnegative when $\lambda$ is large and $A_{\lambda}$ has an odd number of eigenvalues when $\lambda$ tends to 0 . The idea then is to use an infinite determinant to keep track of the sign of the eigenvalues of $A_{\lambda}$ as $\lambda$ varies from 0 to $\infty$.

For the proofs of Theorems 1.2 and 1.3, we carefully use the neutral modes. In the literature, neutral modes have usually been used as the base modes, from which unstable modes have been obtained by the perturbation argument of Tollmien. The novelty of this paper is to utilize a different property of neutral modes: the neutral wave numbers are the possible boundary points of the set of all unstable wave numbers. Thus if we knew all these possible neutral wave numbers and the instability properties around them, we could deduce the stability properties at all the wave numbers. Indeed for our purpose we only need to understand the neutral modes from which the unstable modes can issue. We call them the neutral limiting modes to distinguish them from the usual neutral modes, which are just the solutions to the Rayleigh equation with real $c$.

Definition 1.6. The triple $\left(c_{s}, \alpha_{s}, \phi_{s}\right)$ with $c_{s}$ real and $\alpha_{s}$ positive is said to be a neutral limiting mode if it is the limit of the growing solution sequence $\left(c_{k}, \alpha_{k}, \phi_{k}\right)$ (with $\operatorname{Im} c_{k}>0$ ) of the Rayleigh equation (3). The precise notions of convergence of $\phi_{k}$ to $\phi_{s}$ will be made clear in Lemma 3.6. Formally $\left(c_{s}, \alpha_{s}, \phi_{s}\right)$ ought to satisfy the Rayleigh equation

$$
\left(U-c_{s}\right)\left(\frac{d^{2}}{d y^{2}}-\alpha_{s}^{2}\right) \phi_{s}-U^{\prime \prime} \phi_{s}=0 .
$$

We call $c_{s}$ the neutral limiting phase speed and $\alpha_{s}$ the neutral limiting wave number.

Here in the above definition, the convergence of $\left\{c_{k}\right\}$ is guaranteed by Howard's semicircle theorem (4). From (4) we also know that $c_{s}$ must lie in the range of $U(y)$. The importance of neutral limiting modes lies in the fact that the neutral limiting wave numbers are the possible boundary points of the set of all unstable wave numbers (see Theorem 3.9). The knowledge of the instability near every neutral limiting wave number will allow us to determine the instability in the whole range of wave numbers. 
For that purpose, first we need to know what all the neutral limiting modes are. In general, it is difficult to get a simple answer. But we have the following simple characterization in case the flow is in class $\mathcal{K}$.

THEOREM 1.7. If the flow is in class $\mathcal{K}$, then for any neutral limiting mode $\left(c_{s}, \alpha_{s}, \phi_{s}\right)$ with positive $\alpha_{s}$, the phase speed must be $c_{s}=U_{s}$ and the function $\phi_{s}$ must solve

$$
-\frac{d^{2}}{d y^{2}} \phi_{s}+\frac{U^{\prime \prime}}{U-U_{s}} \phi_{s}=-\alpha_{s}^{2} \phi_{s}
$$

with $\phi_{s}\left(y_{1}\right)=\phi_{s}\left(y_{2}\right)=0$.

In the physics literature [17], [6], for a monotone flow it was shown heuristically by using Reynolds stress that the neutral limiting phase speed must be $U_{s}$. Using some lemmas of Sattinger [22], we rigorously prove the same result for large classes of flows. For flows in class $\mathcal{K}$, we have some uniform a priori bound on the $\mathbf{H}^{2}$ norm of unstable eigenfunctions. This enables us to deduce the other conclusions in the theorem.

Furthermore, for a flow in the class $\mathcal{K}^{+}$, we also obtain the instability property near the neutral wave numbers. This is done by rigorously verifying Tollmien's argument. By combining it with the boundary point property of neutral limiting wave numbers (Theorem 3.9), we obtain an unstable mode for each $\alpha$ in $\left(0, \alpha_{\max }\right)$.

To prove Theorem 1.5, we truncate the unbounded flow to get a sequence of bounded flows. Then by applying Theorems 1.2 and 1.4 to truncated flows, we get a sequence of approximating unstable solutions. We can show that the sequence obtained converges to a nontrivial function, which is an unstable solution to the Rayleigh equation in the unbounded case.

In [1] Bardos, Guo, and Strauss rigorously proved nonlinear instability from the existence of growing modes under a certain assumption for flows defined on bounded domains. For rotating flows as in Theorem 1.3, that assumption is satisfied. For shear flows as in Theorem 1.2, we assume the $x$-direction is $P$-periodic, with the wave number $\alpha$ being multiples of $\frac{2 \pi}{P}$. Then the result in [1] can still apply. The nonlinear instability proved in [1] is in the $\mathbf{L}^{2}$ norm of the vorticity. In [13], Grenier proved nonlinear instability from the existence of growing modes for very general shear flows. In particular, nonlinear instability of shear flows in [13] can be proved in unbounded spaces. Thus the flows in Theorem 1.5 are also nonlinearly unstable. Note that the nonlinear instability in [13] is in the $\mathbf{L}^{\infty}$ and $\mathbf{L}^{2}$ norms of velocity.

We can generalize most of Theorem 1.2 of this paper to general shear flows in the class $\mathcal{F}$ (see Definition 3.1). Thus we can treat any flow with a monotone velocity profile $U(y)$ or any flow that satisfies a differential equation $U^{\prime \prime}(y)=g(U(y)) k(y)$ for some function $k(y)>0$. The details will appear in a forthcoming paper. In [19], we use the method of section 2 to treat linear instability of general ideal plane flows.

The paper is organized as follows. In section 2, we prove Theorem 1.4 for odd flows. We study the neutral limiting modes in section 3. Section 4 is devoted to the proof of Theorem 1.2. In section 5, we give the proof of Theorem 1.3 for the rotating case. We treat unbounded shear flows in section 6 .

2. Odd flows. We divide the proof of Theorem 1.4 into several steps. First we reduce the problem to the eigenvalue problem of an ODE system. Let $c=i \lambda(\lambda>0)$ and $\phi=f+i h$; then (3) becomes

$$
\left(-\frac{d^{2}}{d y^{2}}+\alpha^{2}\right)(f+i h)+\left(\frac{U^{\prime \prime} U}{U^{2}+\lambda^{2}}+i \frac{\lambda U^{\prime \prime}}{U^{2}+\lambda^{2}}\right)(f+i h)=0 .
$$


Comparing the real and imaginary parts of (3) and using the definition of $K(y)$, we get

$$
\begin{aligned}
& -\frac{d^{2}}{d y^{2}} f+\alpha^{2} f-K(y) f+K(y) \frac{\lambda^{2}}{\lambda^{2}+U(y)^{2}} f+K(y) \frac{\lambda U(y)}{\lambda^{2}+U(y)^{2}} h=0, \\
& -\frac{d^{2}}{d y^{2}} h+\alpha^{2} h-K(y) h-K(y) \frac{\lambda U(y)}{\lambda^{2}+U(y)^{2}} f+K(y) \frac{\lambda^{2}}{\lambda^{2}+U(y)^{2}} h=0
\end{aligned}
$$

with $f=h=0$ at $y=-a, a$. If we denote

$$
A_{0}=\left(\begin{array}{cc}
-\frac{d^{2}}{d y^{2}}+\alpha^{2}-K(y) & 0 \\
0 & -\frac{d^{2}}{d y^{2}}+\alpha^{2}-K(y)
\end{array}\right)
$$

and

$$
B_{\lambda}=K(y)\left(\begin{array}{cc}
\frac{\lambda^{2}}{\lambda^{2}+U(y)^{2}} & \frac{\lambda U(y)}{\lambda^{2}+U(y)^{2}} \\
-\frac{\lambda U(y)}{\lambda^{2}+U(y)^{2}} & \frac{\lambda^{2}}{\lambda^{2}+U(y)^{2}}
\end{array}\right),
$$

$A_{\lambda}=A_{0}+B_{\lambda}$. Then (16) becomes

$$
A_{\lambda}\left(\begin{array}{c}
f \\
h
\end{array}\right)=0
$$

The common domain for the operators $A_{\lambda}$ is

$$
\mathcal{H}=\left\{(f, h) \mid f, h \in\left(\mathbf{H}^{2}(-a, a) \cap \mathbf{H}_{0}^{1}(-a, a)\right) \text { and } f \text { odd, } h \text { even }\right\} .
$$

Let

$$
\mathcal{X}=\left\{(f, h) \mid f, h \in \mathbf{L}^{2}(-a, a) \text { with } f \text { odd, } h \text { even }\right\} .
$$

Here $\mathcal{H}, \mathcal{X}$ are complex spaces. Due to the oddness of $U(y), A_{\lambda}: \mathcal{H} \rightarrow \mathcal{X}$. In the following $\|$.$\| denotes the \mathbf{L}^{2}$ norm. We have the following simple characterization of $A_{\lambda}$.

LEMma 2.1. $A_{\lambda}$ is a densely defined closed operator, and for any $\xi$ in its resolvent set $\rho\left(A_{\lambda}\right),\left(\xi-A_{\lambda}\right)^{-1}$ is a trace class operator. The eigenvalues of $A_{\lambda}$ appear in complex conjugate pairs and are all discrete with finite multiplicity.

Proof. Denote

$$
A=\left(\begin{array}{cc}
-\frac{d^{2}}{d y^{2}} & 0 \\
0 & -\frac{d^{2}}{d y^{2}}
\end{array}\right)
$$

with $D(A)=\mathcal{H}$. Then clearly $(\xi-A)^{-1}$ is a trace class operator for any $\xi \in \rho(A)$ and we have

$$
\left\|(A+k)^{-1}\right\| \leq \frac{1}{k}
$$

for any $k>0$. On the other hand, $A_{\lambda}-A$ are uniformly bounded operators, and suppose $\left\|A_{\lambda}-A\right\| \leq M$. We have

$$
A_{\lambda}+k=A+k+A_{\lambda}-A=\left(1+\left(A_{\lambda}-A\right)(A+k)^{-1}\right)(A+k) .
$$


If $M<k$, then $-k \in \rho\left(A_{\lambda}\right)$ and

$$
\left(A_{\lambda}+k\right)^{-1}=(A+k)^{-1}\left(1+\left(A_{\lambda}-A\right)(A+k)^{-1}\right)^{-1} .
$$

This is the multiplication of a bounded operator with a trace class operator, so it is also in trace class. For any $\xi \in \rho\left(A_{\lambda}\right)$, from formula

$$
\left(\xi-A_{\lambda}\right)^{-1}=\left(-k-A_{\lambda}\right)^{-1}+(\xi+k)\left(\xi-A_{\lambda}\right)^{-1}\left(-k-A_{\lambda}\right)^{-1},
$$

we can see that $\left(\xi-A_{\lambda}\right)^{-1}$ is in trace class.

Now the conclusions about the eigenvalues of $A_{\lambda}$ follow from the trace class property just proved and the fact that the coefficients of $A_{\lambda}$ are real.

Now we study the semigroup generated by $-A_{\lambda}$. Notice that $-A_{\lambda}$ is a bounded perturbation of

$$
A=\left(\begin{array}{cc}
\frac{d^{2}}{d y^{2}} & 0 \\
0 & \frac{d^{2}}{d y^{2}}
\end{array}\right),
$$

which generates the diffusion semigroup. Then by the bounded perturbation theorem of semigroups, we know that $-A_{\lambda}$ generates a strongly continuous semigroup. Denote $T_{\lambda}(t)=\exp \left(-t A_{\lambda}\right)$. Then there exists some $C, \omega$ positive (independent of $\lambda$ ) such that

$$
\left\|T_{\lambda}(t)\right\| \leq C e^{\omega t} .
$$

We have the following characterization of $T_{\lambda}(t)$.

Lemma 2.2. For all $t>0, T_{\lambda}(t)$ is in trace class.

Proof. First we claim that $A_{\lambda} T_{\lambda}(t)$ is a bounded operator. Assuming the claim, the theorem follows easily since we have for any $\xi \in \rho(A)$

$$
T_{\lambda}(t)=\left(\xi-A_{\lambda}\right)^{-1}\left(\left(\xi-A_{\lambda}\right) T_{\lambda}(t)\right),
$$

which is the multiplication of a trace class operator with a bounded operator, so it is in trace class.

We shall now prove the claim, which is due to the smoothing effect of $T_{\lambda}(t)$. We need to show only that $A T_{\lambda}(t)$ is bounded. For this purpose we study the evolution equation associated with $T_{\lambda}(t)$.

$$
\begin{aligned}
& \frac{d}{d t} f=\frac{d^{2}}{d y^{2}} f-\alpha^{2} f+K(y) f-K(y) \frac{\lambda^{2}}{\lambda^{2}+U(y)^{2}} f-K(y) \frac{\lambda U(y)}{\lambda^{2}+U(y)^{2}} h, \\
& \frac{d}{d t} h=\frac{d^{2}}{d y^{2}} h-\alpha^{2} h+K(y) h+K(y) \frac{\lambda U(y)}{\lambda^{2}+U(y)^{2}} f-K(y) \frac{\lambda^{2}}{\lambda^{2}+U(y)^{2}} h
\end{aligned}
$$

with $f(0)=f_{0}, h(0)=h_{0}$. Now to show the claim, it suffices to prove

$$
\left\|\frac{d^{2}}{d y^{2}} f(t)\right\|_{2},\left\|\frac{d^{2}}{d y^{2}} h(t)\right\|_{2} \leq C(t)\left(\left\|f_{0}\right\|_{2}+\left\|h_{0}\right\|_{2}\right)
$$

We denote (17) by

$$
\begin{aligned}
& \frac{d}{d t} f=\frac{d^{2}}{d y^{2}} f+R_{1}(f, h), \\
& \frac{d}{d t} h=\frac{d^{2}}{d y^{2}} h+R_{2}(f, h) .
\end{aligned}
$$


Then it is easy to see that

$$
\begin{aligned}
\left\|R_{1}\right\|_{2},\left\|R_{2}\right\|_{2} & \leq C_{0}\left(\|f(t)\|_{2}+\|h(t)\|_{2}\right) \\
& \leq C_{0} C e^{\omega t}\left(\left\|f_{0}\right\|_{2}+\left\|h_{0}\right\|_{2}\right) .
\end{aligned}
$$

So from the regularity theory of the linear parabolic equation, we have

$$
\begin{aligned}
\left\|\frac{d^{2}}{d y^{2}} f(t)\right\|_{2},\left\|\frac{d^{2}}{d y^{2}} h(t)\right\|_{2} & \leq C^{\prime}(t)\left(\|f(t)\|_{2}+\|h(t)\|_{2}+\left\|R_{1}\right\|_{2}+\left\|R_{2}\right\|_{2}\right) \\
& \leq C^{\prime \prime}(t)\left(\left\|f_{0}\right\|_{2}+\left\|h_{0}\right\|_{2}\right) .
\end{aligned}
$$

Thus the claim is proved.

From Lemmas 2.1 and 2.2, we know that the eigenvalues of $A_{\lambda}$ and $T_{\lambda}(t)$ are discrete with finite multiplicity and that

$$
\sigma\left(T_{\lambda}(t)\right) \backslash\{0\}=\exp \left(-t \sigma\left(A_{\lambda}\right)\right) .
$$

Now denote all the distinct eigenvalues of $A_{\lambda}$ (arranged with nondecreasing real part) by $\mu_{1}(\lambda), \mu_{2}(\lambda), \ldots, \mu_{k}(\lambda), \ldots$, with multiplicities $n_{1}, n_{2}, \ldots, n_{k}, \ldots$. We define the infinite determinant of $I d-T_{\lambda}(1)$ as

$$
d(\lambda)=\prod_{k=1}^{\infty}\left(1-\exp \left(-\mu_{k}(\lambda)\right)\right)^{n_{k}} .
$$

Since $T_{\lambda}(1)$ is a trace class operator and $\mu_{k}(\lambda)$ appears in complex conjugate pairs, $d(\lambda)$ is a finite real number. From the definition of $d(\lambda)$, we know that the sign of $d(\lambda)$ is determined only by the number of negative real eigenvalues of $A_{\lambda}$. If this number is odd, then $d(\lambda)$ is negative. And $d(\lambda)$ is positive if the number is even. Here we always assume $A_{\lambda}$ has no kernel, since otherwise we have already obtained a solution to the Rayleigh equation.

We define three sets

$$
S_{-}=\{\lambda>0 \mid d(\lambda)<0\}, \quad S_{+}=\{\lambda>0 \mid d(\lambda)>0\}, \quad S_{0}=\{\lambda>0 \mid d(\lambda)=0\} .
$$

We will show that $S_{-}, S_{+}$are nonempty open sets. Then the theorem follows easily, as we shall now show.

Proof of Theorem 1.4. We claim that $S_{0}$ is nonempty. Otherwise we would have $(0,+\infty)=S_{-} \cup S_{+}$, which is impossible, since $S_{-}, S_{+}$are two disjoint open sets. So there must exist some $\lambda_{0}>0$ such that $d\left(\lambda_{0}\right)=0$. Then there exists $k$ so that $1-\exp \left(-\mu_{k}\left(\lambda_{0}\right)\right)=0$. So $\mu_{k}\left(\lambda_{0}\right)=0$ and $A_{\lambda_{0}}$ has a nontrivial kernel $(f, h)$. This means that $c=i \lambda_{0}, \phi=f+i h$ is a solution to Rayleigh's equation (3).

The next several lemmas prove the properties of $S_{-}, S_{+}$that we need.

Lemma 2.3. $S_{+}$is nonempty.

Proof. Because for any real vector $(f, h)$,

$$
\begin{aligned}
\left((f, h), A_{\lambda}\left(\begin{array}{c}
f \\
h
\end{array}\right)\right)= & \left(\left(-\frac{d^{2}}{d y^{2}}+\alpha^{2}-K(y)+K(y) \frac{\lambda^{2}}{\lambda^{2}+U(y)^{2}}\right) f, f\right) \\
& +\left(\left(-\frac{d^{2}}{d y^{2}}+\alpha^{2}-K(y)+K(y) \frac{\lambda^{2}}{\lambda^{2}+U(y)^{2}}\right) h, h\right) \\
& >0
\end{aligned}
$$


when $\lambda$ is large, $A_{\lambda}$ is a positive operator. Thus all its real eigenvalues are positive, so that $d(\lambda)>0$ for $\lambda$ large.

LEMma 2.4. $S_{-}$is nonempty.

Proof. From the assumptions of Theorem 1.4 and the definition of operator $A_{0}$, we know that $d(0)<0$. We will show that for $\lambda$ small, $d(\lambda)<0$.

First we claim that

(i) for any eigenvalue $\mu(\lambda)$ of $A_{\lambda}$, we have $|\operatorname{Im} \mu(\lambda)|<\|K\|_{\infty}$;

(ii) there exists positive $\varepsilon_{1}, \delta_{1}$ such that if $0 \leq \lambda<\delta_{1}$, then for any eigenvalue $\mu(\lambda)$ of $A_{\lambda}$, we have $|\operatorname{Re} \mu(\lambda)|>\varepsilon_{1}$.

Proof of claim (i). Let $(f, h)$ be the eigenfunction with $\|f\|_{2}+\|h\|_{2}=1$. Taking inner products with the conjugate $(\bar{f}, \bar{h})$ on both sides of

$$
A_{\lambda}\left(\begin{array}{c}
f \\
h
\end{array}\right)=\mu(\lambda)\left(\begin{array}{l}
f \\
h
\end{array}\right)
$$

and comparing the imaginary parts, we get

$$
\begin{aligned}
|\operatorname{Im} \mu(\lambda)| & \leq\left|2\|K\|_{\infty} \operatorname{Im} \int_{-a}^{a} \frac{\lambda U(y)}{\lambda^{2}+U(y)^{2}} f \bar{h} d y\right| \\
& \leq\|K\|_{\infty} \frac{1}{2}\left(\|f\|_{2}+\|h\|_{2}\right)^{2}=\frac{1}{2}\|K\|_{\infty} .
\end{aligned}
$$

Proof of claim (ii). Supposing it is not true, we could find a sequence $\lambda_{n} \rightarrow 0$, $\mu_{n}$ being an eigenvalue of $A_{\lambda_{n}}$, and $\operatorname{Re} \mu_{n} \rightarrow 0$. Let $\left(f_{n}, h_{n}\right)$ be the corresponding eigenfunction and $\left\|f_{n}\right\|_{2}+\left\|h_{n}\right\|_{2}=1$. By (i), $\left\{\mu_{n}\right\}$ is a bounded sequence. We can find a subsequence such that $\mu_{n_{k}} \rightarrow \mu_{0}$, so that $\mu_{0}$ is purely imaginary. We still denote the subsequence by $\left\{\mu_{n}\right\}$.

From the equation satisfied by the eigenfunction $\left(f_{n}, h_{n}\right)$, we get

$$
\left\|f_{n}\right\|_{\mathbf{H}^{2}},\left\|g_{n}\right\|_{\mathbf{H}^{2}} \leq C\left(\|f\|_{2}+\|h\|_{2}\right)=C
$$

from elliptic regularity theory by noticing that the coefficients in (19) are uniformly bounded. Thus there exists a subsequence such that $\left(f_{n_{k}}, g_{n_{k}}\right) \rightarrow\left(f_{0}, g_{0}\right)$ weakly in $\mathbf{H}^{2}$ and strongly in $\mathbf{H}^{1}$. Moreover,

$$
\begin{aligned}
\left\|\left(A_{0}-\mu_{0}\right)\left(\begin{array}{c}
f_{n_{k}} \\
h_{n_{k}}
\end{array}\right)\right\| & \leq\|K\|_{\infty}\left(\left\|\frac{\lambda_{n_{k}}^{2}}{\lambda_{n_{k}}^{2}+U(y)^{2}}\right\|_{2}+\left\|\frac{\lambda_{n_{k}} U(y)}{\lambda_{n_{k}}^{2}+U(y)^{2}}\right\|_{2}\right) \\
& \times\left(\left\|f_{n_{k}}\right\|_{\infty}+\left\|h_{n_{k}}\right\|_{\infty}\right)+\left|\mu_{n_{k}}-\mu_{0}\right|\left(\left\|f_{n_{k}}\right\|_{2}+\left\|h_{n_{k}}\right\|_{2}\right) \\
& \leq C\left(\left\|\frac{\lambda_{n_{k}}^{2}}{\lambda_{n_{k}}^{2}+U(y)^{2}}\right\|_{2}+\left\|\frac{\lambda_{n_{k}} U(y)}{\lambda_{n_{k}}^{2}+U(y)^{2}}\right\|_{2}+\left|\mu_{n_{k}}-\mu_{0}\right|\right)
\end{aligned}
$$

tends to zero as $\lambda_{n_{k}} \rightarrow 0$. Thus we have $\mu_{0} \in \sigma\left(A_{0}\right)$, which is a contradiction to the fact that $A_{0}$ has no eigenvalue lying on the imaginary axis. So claim (ii) is proved.

Let $\Lambda$ be the infimum of real part of eigenvalues of $A_{\lambda} . \Lambda$ is finite since $A_{\lambda}$ is uniformly bounded from below. Define

$$
D=\left\{(x, y) \mid \Lambda-1<x<-\frac{\varepsilon_{1}}{2},-\|K\|_{\infty}<y<\|K\|_{\infty}\right\}
$$


and $\Gamma=\partial D$. From claim (ii), if $\lambda<\delta_{1}$, all eigenvalues of $A_{\lambda}$ with negative real part lie in $D$. Define the Riesz projection as

$$
P_{\lambda}=\frac{1}{2 \pi i} \oint_{\Gamma}\left(k-A_{\lambda}\right)^{-1} d k
$$

and $R\left(P_{\lambda}\right)$ its range, where $\lambda \geq 0$ and the $\Gamma$-integral is in the counterclockwise sense. Then by the definition of $d(\lambda)$

$$
\operatorname{sign} d(\lambda)=(-1)^{\operatorname{dim} R\left(P_{\lambda}\right)} .
$$

To prove the lemma, it suffices to show that $\left\|P_{\lambda}-P_{0}\right\| \rightarrow 0$ as $\lambda \rightarrow 0$. If so, then $\operatorname{dim}\left(R\left(P_{\lambda}\right)\right)=\operatorname{dim}\left(R\left(P_{0}\right)\right)$ if $\lambda$ is small enough. By the definition of $P_{0}, \operatorname{dim}\left(R\left(P_{0}\right)\right)$ is the number of negative eigenvalues of $A_{0}$ on the space $\mathcal{H}$, which is equal to that of the operator $-\frac{d^{2}}{d y^{2}}+\alpha^{2}-K(y)$ on the space $\mathbf{H}^{2}(-a, a) \cap \mathbf{H}_{0}^{1}(-a, a)$. This is due to the fact that any eigenfunction of $-\frac{d^{2}}{d y^{2}}+\alpha^{2}-K(y)$ is either odd or even when $K(y)$ is even. With $\alpha$ lying in the intervals of Theorem 1.4, $-\frac{d^{2}}{d y^{2}}+\alpha^{2}-K(y)$ has an odd number of negative eigenvalues, so $\operatorname{dim}\left(R\left(P_{0}\right)\right)$ is odd. Thus when $\lambda$ is small enough, $\operatorname{dim}\left(R\left(P_{\lambda}\right)\right)$ is odd, which implies that $d(\lambda)$ is negative by (21) so that $S_{-}$ is not empty.

To show $\left\|P_{\lambda}-P_{0}\right\| \rightarrow 0$, we note that

$$
\begin{aligned}
& \left\|B_{\lambda}\left(\begin{array}{c}
f \\
h
\end{array}\right)\right\| \leq\|K\|_{\infty}\left(\left\|\frac{\lambda^{2}}{\lambda^{2}+U(y)^{2}}\right\|_{2}+\left\|\frac{\lambda U(y)}{\lambda^{2}+U(y)^{2}}\right\|_{2}\right)\left(\|f\|_{\infty}+\|h\|_{\infty}\right) \\
& \leq C\|K\|_{\infty}\left(\left\|\frac{\lambda^{2}}{\lambda^{2}+U(y)^{2}}\right\|_{2}+\left\|\frac{\lambda U(y)}{\lambda^{2}+U(y)^{2}}\right\|_{2}\right)\left(\left\|A_{0}\left(\begin{array}{c}
f \\
h
\end{array}\right)\right\|+\left\|\left(\begin{array}{c}
f \\
h
\end{array}\right)\right\|\right) \\
& =C(\lambda)\left(\left\|A_{0}\left(\begin{array}{c}
f \\
h
\end{array}\right)\right\|+\left\|\left(\begin{array}{c}
f \\
h
\end{array}\right)\right\|\right),
\end{aligned}
$$

where

$$
C(\lambda)=C\|K\|_{\infty}\left(\left\|\frac{\lambda^{2}}{\lambda^{2}+U(y)^{2}}\right\|_{2}+\left\|\frac{\lambda U(y)}{\lambda^{2}+U(y)^{2}}\right\|_{2}\right) \rightarrow 0
$$

as $\lambda \rightarrow 0$ by dominant convergence. Since $\Gamma \subset \sigma\left(A_{\lambda}\right)$ if $\lambda<\delta_{1}$ and $\Gamma$ is compact, it follows that $\left\|\left(\xi-A_{\lambda}\right)^{-1}\right\|$ is uniformly bounded by some constant $M$ independent of $\xi \in \Gamma$. Then we have

$$
\begin{aligned}
\left\|\left(\xi-A_{\lambda}\right)^{-1}-\left(\xi-A_{0}\right)^{-1}\right\| & =\left\|\left(\xi-A_{\lambda}\right)^{-1} B_{\lambda}\left(\xi-A_{0}\right)^{-1}\right\| \\
& \leq\left\|\left(\xi-A_{\lambda}\right)^{-1}\right\|\left\|B_{\lambda}\left(\xi-A_{0}\right)^{-1}\right\| \\
& \leq M C(\lambda)\left(\left\|A_{0}\left(\xi-A_{0}\right)^{-1}\right\|+\left\|\left(\xi-A_{0}\right)^{-1}\right\|\right) \\
& \leq M C(\lambda)\left(1+\left\|\xi\left(\xi-A_{0}\right)^{-1}\right\|+\left\|\left(\xi-A_{0}\right)^{-1}\right\|\right) .
\end{aligned}
$$

So as $\lambda \rightarrow 0,\left\|\left(\xi-A_{\lambda}\right)^{-1}-\left(\xi-A_{0}\right)^{-1}\right\| \rightarrow 0$ uniformly for $\xi \in \Gamma$. Thus $\left\|P_{\lambda}-P_{0}\right\| \rightarrow$ 0 if $\lambda \rightarrow 0$. 
Lemma 2.5. $S_{-}$and $S_{+}$are open sets.

Proof. We will show that $S_{-}$is open. The proof for $S_{+}$is the same. Suppose $\lambda_{0} \in S_{-}$. Let $b>0$ be such that there is no eigenvalue of $A_{\lambda_{0}}$ with real part $b$. Then by the same argument as in the last lemma, there exists $\varepsilon_{1}, \delta_{1}>0$ such that if $\left|\lambda-\lambda_{0}\right|<\delta_{1}$, then for any eigenvalue $\mu(\lambda)$ of $A_{\lambda}$, we have $|\operatorname{Re} \mu(\lambda)-b|>\varepsilon_{1}$. Let $\Lambda$ be the infimum of real part of eigenvalues of $A_{\lambda}$. Define

$$
D=\left\{(x, y) \mid \Lambda-1<x<-\frac{\varepsilon_{1}}{2}+b,-\|K\|_{\infty}<y<\|K\|_{\infty}\right\}
$$

and $\Gamma=\partial D$. Then all eigenvalues of $A_{\lambda}$ with real part smaller than $b$ lie in $D$ and $\Gamma \subset \sigma\left(A_{\lambda}\right)$ provided $\left|\lambda-\lambda_{0}\right|<\delta_{1}$. Define $P_{\lambda}$ by (20). Then $\left\|P_{\lambda}-P_{\lambda_{0}}\right\| \rightarrow 0$ as $\left|\lambda-\lambda_{0}\right| \rightarrow 0$, since $A_{\lambda}$ is analytic for $\lambda>0$. So $\operatorname{dim}\left(R\left(P_{\lambda}\right)\right)=\operatorname{dim}\left(R\left(P_{\lambda_{0}}\right)\right)$ if $\left|\lambda-\lambda_{0}\right|$ is small enough. Let $\mu_{1}, \mu_{2}, \ldots, \mu_{N}$ be all the distinct eigenvalues of $A_{\lambda_{0}}$ in $D$. Let $m_{k}$ be the multiplicity of $\mu_{k}$. Now for each $\mu_{k}$, we can take a small ball $B_{k}=B\left(\mu_{k} ; r_{k}\right)$ such that there are no other eigenvalues of $A_{\lambda_{0}}$ in it besides $\mu_{k}$. And by taking $r_{k}$ small enough we can suppose that $B_{k}$ does not intersect with the imaginary axis if $\operatorname{Re} \mu_{k} \neq 0$, and $B_{k}$ does not intersect with the real axis if $\operatorname{Re} \mu_{k}=0$. Also $B_{k}$ does not intersect with $\Gamma$. They are disjoint with others, and for the conjugate eigenvalue we take the same radius. Then if $\left|\lambda-\lambda_{0}\right|$ is small enough, by analytic perturbation theory, there are exactly $m_{k}$ eigenvalues (counting multiplicity) of $A_{\lambda}$ in each $B_{k}$. Since $\operatorname{dim}\left(R\left(P_{\lambda}\right)\right)=\operatorname{dim}\left(R\left(P_{\lambda_{0}}\right)\right)$, these are all the eigenvalues of $A_{\lambda}$ in $D$. Now notice that for each $B_{k}$ and its conjugate one, if we multiply all the eigenvalues of $A_{\lambda}$ in them, the sign is the same as for $A_{\lambda_{0}}$. So in the definition of $d(\lambda)$, the part corresponding to the multiplication of all eigenvalues of $A_{\lambda}$ with real part smaller than $b$ is of the same sign with the $\lambda_{0}$ case. Thus it is negative if $\left|\lambda-\lambda_{0}\right|$ is small. While the other part of multiplication is always positive, we proved that $d(\lambda)$ is negative when $\left|\lambda-\lambda_{0}\right|$ is small. This finishes the proof of the lemma.

It is easy to see that we can get the following abstract version by the same proof.

Theorem 2.6. Consider a family of real operators $A_{\lambda}=-A+B_{\lambda}(\lambda \in(0,+\infty))$ with the same domain $\mathcal{H}$. We assume the following:

(I) $B_{\lambda}$ is bounded and norm continuous for positive $\lambda$.

(II) A generates a generalized parabolic semigroup; that is, $\exp (t A)$ is in trace class and $A \exp (t A)$ is bounded.

(III) When $\lambda$ is sufficiently large, $A_{\lambda}$ has no eigenvalue with negative real part.

(IV) When $\lambda$ tends to $0, A_{\lambda}$ tend to $A_{0}$ in the sense that

$$
\left\|\left(A_{\lambda}-A_{0}\right) \phi\right\| \leq c(\lambda)\left(\left\|A_{0} \phi\right\|+\|\phi\|\right),
$$

$c(\lambda) \rightarrow 0$ as $\lambda \rightarrow 0^{+}$for any function $\phi \in \mathcal{H}$. Then if $A_{0}$ has an odd number of negative eigenvalues and no kernel, there must exist some $\lambda_{0}>0$ such that $A_{\lambda_{0}}$ has a nontrivial kernel.

We can also treat the periodic and Neumann boundary conditions for the Rayleigh equation by the same method. The conclusion and the proofs are direct analogues of Theorem 1.4.

Example 2.7 (doubly symmetric flows). Theorem 1.4 could be used to treat some nonodd flows.

Suppose that $U(y)$ is even on $(0,2 d)$ with respect to its midpoint $d$ and is odd on $(0, d)$ with respect to its midpoint $\frac{d}{2}$. In that case, we could treat the sinuous (even mode) and varicose (odd mode) separately by studying the Rayleigh equation on $[0, d]$, taking the boundary condition at $d$ to be either $\phi^{\prime}(d)=0$ or $\phi(d)=0$. We could treat the varicose case by Theorem 1.4. 


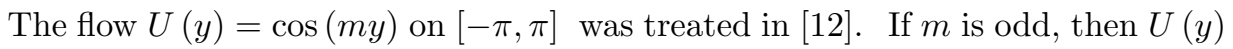
is in the class we described above. For varicose modes, we can restrict the problem to $[0, \pi]$ and furthermore restrict the function space to be the space $\mathcal{P}_{j}$ spanned by $\sin n y$ $(n=j+m p)$. Here $j$ is a fixed integer in $[1,[m / 2]]$. Then the space $\mathcal{P}_{j}$ is invariant under the operator $A_{\lambda}$ corresponding to $U(y)=\cos (m y)$. Notice that if

$$
m^{2}-(m-j)^{2}<\alpha^{2}<m^{2}-j^{2},
$$

then $-\frac{d^{2}}{d y^{2}}+\alpha^{2}-m^{2}$ has only one negative eigenvalue on $\mathcal{P}_{j}$. Thus from Theorem 1.4, we know that there is a purely growing unstable mode. This was proved in [12] by a continued fractions technique. It was also shown in [12] by numerical computation that if $\alpha$ is small, there is no purely growing mode.

3. Neutral limiting modes. In this section, we study properties of the possible neutral limiting modes. For a certain class of flows, we get a simple characterization of the neutral limiting phase speed $c_{s}$. For flows of class $\mathcal{K}$, we get a complete characterization as in Theorem 1.7.

Definition 3.1. A velocity profile $U(y)$ is said to be in class $\mathcal{F}$ if for each number $c$ in the range of $U$ but not an inflection value, $U^{\prime \prime}$ takes the same sign at all points where $U(y)=c$.

Some examples in class $\mathcal{F}$ are a monotone flow, a symmetric flow with monotone half part, and a flow satisfying $U^{\prime \prime}(y)=g(U(y)) k(y)$ for some function $g$ and $k(y)>$ 0 . It is readily seen that $\mathcal{K} \subset \mathcal{F}$.

Remark 3.2. We mention two simple facts we will use later.

(i) For a $\mathbf{C}^{2}$ flow $U(y)$, if $c$ is not an inflection value, then $U(y)=c$ can only hold at a finite number of points.

(ii) For a $\mathbf{C}^{2}$ flow $U(y)$, if there exists some inflection value $U_{s}$ such that the function

$$
K(y):=-U^{\prime \prime}(y) /\left(U(y)-U_{s}\right)
$$

is bounded on $\left[y_{1}, y_{2}\right]$, then $U(y)-U_{s}=0$ can only hold at a finite number of points.

For the proof of (i), we notice that $U^{\prime \prime}\left(y_{0}\right) \neq 0$ at any point $y_{0} \in\{U(y)=c\}$, since $c$ is not an inflection value. So $y_{0}$ is an isolated point of $\{U(y)=c\}$. Therefore $\{U(y)=c\}$ is a finite set. For (ii) we observe that $\phi=U(y)-U_{s}$ solves a second order regular ODE

$$
\phi^{\prime \prime}+K(y) \phi=0
$$

on $\left[y_{1}, y_{2}\right]$. So the zeros of $\phi$ cannot cluster in the interval.

TheOREM 3.3. If $U(y)$ is in class $\mathcal{F}$, then the neutral limiting phase speed must be an inflection value.

Note that in Definition 1.6, $\alpha_{s}$ is positive. If $\alpha_{s}=0$, then the neutral limiting phase speed might not be the inflection value. A counterexample is $U(y)=\cos (6 y)$, $y \in(-\pi, \pi)$. The numerical computation in [12] indicated that when $\alpha_{s}=0$, the neutral limiting phase speed is $c_{s}=-1$ while the inflection value is 0 .

For the proof of this theorem, we need several lemmas from the literature, which we state without proof. The first one is an important equality which was first used to prove Rayleigh's criterion. 
LEMma 3.4. Let $\phi$ be a solution of (3) with complex eigenvalue $c=c_{r}+i c_{i}$ $\left(c_{i} \neq 0\right)$, and let

$$
J_{q}(\phi)=\int_{y_{1}}^{y_{2}}\left(\left|\phi^{\prime}\right|^{2}+\alpha^{2}|\phi|^{2}+\frac{U^{\prime \prime}(U-q)}{|U-c|^{2}}|\phi|^{2}\right) d y .
$$

Then $J_{q}(\phi)=0$ for every real number $q$.

Proof. We multiply the Rayleigh equation

$$
\left(\frac{d^{2}}{d y^{2}}-\alpha^{2}\right) \phi-\frac{U^{\prime \prime}}{U-c} \phi=0
$$

by $\phi^{*}(*$ denotes the complex conjugate) and integrate it to get

$$
\int_{y_{1}}^{y_{2}}\left(\left|\phi^{\prime}\right|^{2}+\alpha^{2}|\phi|^{2}+\frac{U^{\prime \prime}}{U-c}|\phi|^{2}\right) d y=0 .
$$

Comparing real and imaginary parts, we get

$$
\begin{gathered}
\int_{y_{1}}^{y_{2}}\left(\left|\phi^{\prime}\right|^{2}+\alpha^{2}|\phi|^{2}+\frac{U^{\prime \prime}\left(U-c_{r}\right)}{|U-c|^{2}}|\phi|^{2}\right) d y=0, \\
\int_{y_{1}}^{y_{2}} \frac{U^{\prime \prime}}{|U-c|^{2}}|\phi|^{2} d y=0 .
\end{gathered}
$$

Combining (24) and (25), we get the conclusion.

We also need some results from [22]. In the following we use the notation in [22]. Let $c$ be any real number in the range of $U(y)$ and let $z_{1}<z_{2}<\cdots<z_{k_{c}}$ be the zeros of $U(y)-c$. Here we assume $k_{c}$ is finite. In the following we always consider the cases in Remark 3.2, so this assumption is valid. We denote by $S_{0}$ the complement of the set of points $\left\{z_{i}\right\}$ in the interval $\left[y_{1}, y_{2}\right]$. Let $z_{0}=y_{1}$ and $z_{k_{c}+1}=y_{2}$. Then we have the following lemma.

Lemma 3.5. Let $\phi$ satisfy (3) with positive $\alpha$ and $c$ as above on $S_{0}$, where $\phi$ is sectionally continuous on the open intervals $\left(z_{j}, z_{j+1}\right), j=0,1, \ldots, k_{c}$. Then $\phi$ cannot vanish at both endpoints of any of the intervals $\left(z_{j}, z_{j+1}\right)$ unless it vanishes identically on that interval.

Proof. This lemma was proved in [22], where it was used for a different purpose, namely, to show that for a fixed wave number there are only a finite number of unstable eigenvalues of the Rayleigh equation under some conditions. Here we give the proof for completeness.

The Rayleigh equation (3) can be rewritten as

$$
\left((U-c) \phi^{\prime}-U^{\prime} \phi\right)^{\prime}=\alpha^{2}(U-c) \phi .
$$

Suppose $\phi\left(z_{i}+\right)=\phi\left(z_{i+1}-\right)=0$ and study (26) in $\left[z_{i}, z_{i+1}\right]$. From the definition of $z_{i}, U-c$ has constant sign in $\left(z_{i}, z_{i+1}\right)$.

If $z_{i} \neq y_{1}(i \neq 0)$, then $U\left(z_{i}\right)-c=0$. Let $\tilde{z} \leq z_{i+1}$ be the nearest zero of $\phi$ in $\left(z_{i}, z_{i+1}\right]$. Since $(26)$ is a real equation, we may assume $\phi$ is real and nonnegative on the interval $\left(z_{i}, \tilde{z}\right)$ and that $\phi^{\prime}\left(z_{i}\right) \geq 0$ and $\phi^{\prime}(\tilde{z}) \leq 0$. Integrating (26) over $\left(z_{i}, \tilde{z}\right)$, we get

$$
(U(\tilde{z})-c) \phi^{\prime}(\tilde{z})=\alpha^{2} \int_{z_{i}}^{\tilde{z}} \phi(U-c) d z^{\prime}
$$

since $\phi$ vanishes at the endpoints $z_{i}, \tilde{z}$. 
If $\tilde{z}=z_{i+1}$, then the left-hand side above must be zero. Hence $\phi$ is identically zero on $\left(z_{i}, z_{i+1}\right)$. On the other hand, if $\tilde{z}<z_{i+1}$, then $U(\tilde{z}) \neq c$ and

$$
\phi^{\prime}(\tilde{z})=\alpha^{2} \int_{z_{i}}^{\tilde{z}} \frac{\left(U\left(z^{\prime}\right)-c\right)}{(U(\tilde{z})-c)} \phi\left(z^{\prime}\right) d z^{\prime},
$$

which could not hold true unless $\phi \equiv 0$ on $\left[z_{i}, \tilde{z}\right]$. But the second order ODE (26) is regular on $\left(z_{i}, z_{i+1}\right)$. Thus $z$ could not be a cluster point of a nontrivial solution $\phi$. Thus $\phi$ must be identically zero on $\left(z_{i}, z_{i+1}\right)$.

If $i=0$, then we repeat the same argument with the right endpoint of the interval $\left(y_{1}, z_{1}\right)$.

Lemma 3.6. Let $\left\{\left(c_{k}, \alpha_{k}, \phi_{k}\right)\right.$ (with $\left.\left.\operatorname{Im} c_{k}>0\right)\right\}_{k=1}^{\infty}$ be the solutions to the Rayleigh equation (3) and $\left\|\phi_{k}\right\|=1$, and $\left(c_{k}, \alpha_{k}\right)$ converges to $\left(c_{s}, \alpha_{s}\right)$ with positive $\alpha_{s}$. Then $\phi_{k}$ converges uniformly to a function $\phi_{s}$ on any compact subset of $S_{0}, \phi_{s}^{\prime \prime}$ exists on $S_{0}$, and $\phi_{s}$ satisfies (14).

The case when $\alpha_{k}$ is independent of $k$ was proved in [22], but the proof can be applied to the current case without much change. The basic idea is that on compact subsets of $S_{0}$, the function $1 /\left(U(y)-c_{k}\right)$ is uniformly bounded, so we get a uniform bound on the derivatives of $\phi_{k}$ up to second order.

Proof of Theorem 3.3. Let $\left(c_{s}, \alpha_{s}, \phi_{s}\right)$ be a neutral limiting mode and assume $c_{s}$ is not an inflection value. First we show that the $\phi_{s}$ obtained by Lemma 3.6 is not identically zero. Otherwise suppose $\phi_{s} \equiv 0$. Let $z_{1}, z_{2}, \ldots, z_{m}$ be all the zeros of $U(y)-c_{s}$, which by Remark 3.2 is finite. Then by the assumption of the theorem and the definition of class $\mathcal{F}$, all $U^{\prime \prime}\left(z_{i}\right)$ have the same sign, say positive. Let $E_{\delta}=$ $\left\{y \in\left[y_{1}, y_{2}\right]|| y-z_{i} \mid<\delta\right.$ for some $\left.i\right\}$. Then $E_{\delta}^{c} \subset S_{0}$ and $U^{\prime \prime}(y)>0$ for $y \in E_{\delta}$ if $\delta$ small enough. Take $q=\min U(y)-1$ and assume $\left\|\phi_{k}\right\|_{2}=1$. Then

$$
\begin{aligned}
J_{q}\left(\phi_{k}\right) & =\int_{y_{1}}^{y_{2}}\left(\left|\phi_{k}^{\prime}\right|^{2}+\alpha_{k}^{2}\left|\phi_{k}\right|^{2}+\frac{U^{\prime \prime}(U-q)}{\left|U-c_{k}\right|^{2}}\left|\phi_{k}\right|^{2}\right) d y \\
& \geq \alpha_{k}^{2}+\int_{E_{\delta}^{c}} \frac{U^{\prime \prime}(U-q)}{\left|U-c_{k}\right|^{2}}\left|\phi_{k}\right|^{2} d y+\int_{E_{\delta}} \frac{U^{\prime \prime}(U-q)}{\left|U-c_{k}\right|^{2}}\left|\phi_{k}\right|^{2} d y \\
& \geq \alpha_{k}^{2}-\sup _{E_{\delta}^{c}} \frac{\left|U^{\prime \prime}(U-q)\right|}{\left(U-c_{k}\right)^{2}} \int_{E_{\delta}^{c}}\left|\phi_{k}\right|^{2} d y .
\end{aligned}
$$

Since $\phi_{k}$ converges to $\phi_{s} \equiv 0$ uniformly on $E_{\delta}^{c}$, we have

$$
\lim _{k \rightarrow \infty} \inf J_{q}\left(\phi_{k}\right) \geq \alpha_{s}^{2} .
$$

So for large $k, J_{q}\left(\phi_{k}\right) \neq 0$, which is a contradiction to Lemma 3.4.

So by Lemma 3.5, there is some $z_{i}$ such that $\phi_{s}\left(z_{i}\right) \neq 0$. Then

$$
\int_{E_{\delta}} \frac{U^{\prime \prime}(U-q)}{\left|U-c_{s}\right|^{2}}\left|\phi_{s}\right|^{2} d y \geq \int_{\left|y-z_{i}\right|<\delta} \frac{U^{\prime \prime}}{\left|U-c_{s}\right|^{2}}\left|\phi_{s}\right|^{2} d y=+\infty
$$

since $c_{s}$ is not an inflection value. By Fatou's lemma,

$$
\lim _{k \rightarrow \infty} \inf \int_{E_{\delta}} \frac{U^{\prime \prime}(U-q)}{\left|U-c_{k}\right|^{2}}\left|\phi_{k}\right|^{2} d y=+\infty
$$

So from

$$
J_{q}\left(\phi_{k}\right) \geq \int_{E_{\delta}} \frac{U^{\prime \prime}(U-q)}{\left|U-c_{k}\right|^{2}}\left|\phi_{k}\right|^{2} d y-\sup _{E_{\delta}^{c}} \frac{U^{\prime \prime}(U-q)}{\left|U-c_{k}\right|^{2}},
$$


we get $\lim _{k} \inf J_{q}\left(\phi_{k}\right)=+\infty$, which is a contradiction to the fact that $J_{q}\left(\phi_{k}\right)=0$ (Lemma 3.4). Thus $c_{s}$ must be an inflection value. This ends the proof of Theorem 3.3 .

To show Theorem 1.7, we need to get some a priori estimate for the sequence of unstable solutions $\left\{\phi_{k}\right\}$ in Definition 1.6. We have the following.

Lemma 3.7. For the flow $U(y)$ in class $\mathcal{K}$, if $\phi$ is the solution to (3) with $\operatorname{Im} c>0$, then we have

$$
\int_{y_{1}}^{y_{2}}\left(\left|\phi^{\prime}\right|^{2}+\alpha^{2}|\phi|^{2}\right) d y<\int_{y_{1}}^{y_{2}} K(y)|\phi|^{2} d y
$$

and

$$
\int_{y_{1}}^{y_{2}}\left(\left|\phi^{\prime \prime}\right|^{2}+2 \alpha^{2}\left|\phi^{\prime}\right|^{2}+\alpha^{4}|\phi|^{2}\right) d y<\|K\|_{\infty} \int_{y_{1}}^{y_{2}} K(y)|\phi|^{2} d y
$$

Proof. Inequality (27) was obtained in [8], but we prove it here for completeness. Denote $c=c_{r}+i c_{i}\left(c_{i}>0\right)$. By Lemma 3.4, for any real $q$

$$
\int_{y_{1}}^{y_{2}}\left(\left|\phi^{\prime}\right|^{2}+\alpha^{2}|\phi|^{2}+\frac{U^{\prime \prime}(U-q)}{\left|U-c_{r}\right|^{2}+c_{i}^{2}}|\phi|^{2}\right) d y=0 .
$$

Let $q=U_{s}-2\left(U_{s}-c_{r}\right)$. Then

$$
\begin{aligned}
\int_{y_{1}}^{y_{2}}\left(\left|\phi^{\prime}\right|^{2}+\alpha^{2}|\phi|^{2}\right) d y & =\int_{y_{1}}^{y_{2}} K(y) \frac{\left(U-U_{s}\right)(U-q)}{\left|U-c_{r}\right|^{2}+c_{i}^{2}}|\phi|^{2} d y \\
& =\int_{y_{1}}^{y_{2}} K(y) \frac{\left(U-U_{s}\right)^{2}+2\left(U-U_{s}\right)\left(U_{s}-c_{r}\right)}{\left|U-c_{r}\right|^{2}+c_{i}^{2}}|\phi|^{2} d y \\
& =\int_{y_{1}}^{y_{2}} K(y) \frac{\left(U-c_{r}\right)^{2}-\left(U_{s}-c_{r}\right)^{2}}{\left|U-c_{r}\right|^{2}+c_{i}^{2}}|\phi|^{2} d y \\
& <\int_{y_{1}}^{y_{2}} K(y)|\phi|^{2} d y .
\end{aligned}
$$

This proves $(27)$.

In (29), let $q=U_{s}$, we get by $(27)$

$$
\int_{y_{1}}^{y_{2}} K(y) \frac{\left(U-U_{s}\right)^{2}}{\left|U-c_{r}\right|^{2}+c_{i}^{2}}|\phi|^{2} d y=\int_{y_{1}}^{y_{2}}\left(\left|\phi^{\prime}\right|^{2}+\alpha^{2}|\phi|^{2}\right) d y<\int_{y_{1}}^{y_{2}} K(y)|\phi|^{2} d y .
$$

We shall show that

$$
\int_{y_{1}}^{y_{2}}\left(\left|\phi^{\prime \prime}\right|^{2}+2 \alpha^{2}\left|\phi^{\prime}\right|^{2}+\alpha^{4}|\phi|^{2}\right) d y-\int_{y_{1}}^{y_{2}} \frac{\left(U^{\prime \prime}\right)^{2}|\phi|^{2}}{\left|U-c_{r}\right|^{2}+c_{i}^{2}} d y=0
$$

which was first proved in [2]. For completeness we now give the proof of (31). We multiply the Rayleigh equation

$$
\left(\frac{d^{2}}{d y^{2}}-\alpha^{2}\right) \phi-\frac{U^{\prime \prime}}{U-c} \phi=0
$$


by $\left(\phi^{*}\right)^{\prime \prime}$ and integrate it to get

$$
\int_{y_{1}}^{y_{2}}\left(\left(\phi^{*}\right)^{\prime \prime}\left(\phi^{\prime \prime}-\alpha^{2} \phi\right)\right)=\int_{y_{1}}^{y_{2}}\left(\left(\phi^{*}\right)^{\prime \prime} \frac{U^{\prime \prime}}{U-c} \phi\right) .
$$

By integration by parts,

$$
\text { LHS of }(32)=\int_{y_{1}}^{y_{2}}\left(\left|\phi^{\prime \prime}\right|^{2}+\alpha^{2}\left|\phi^{\prime}\right|^{2}\right) d y .
$$

Using the Rayleigh equation for $\phi^{*}$, we have

$$
\begin{aligned}
R H S \text { of }(32) & =\int_{y_{1}}^{y_{2}}\left(\left(\alpha^{2} \phi^{*}+\left(\frac{U^{\prime \prime}}{U-c} \phi\right)^{*}\right)\left(\frac{U^{\prime \prime}}{U-c} \phi\right)\right) d y \\
& =\alpha^{2} \int_{y_{1}}^{y_{2}} \frac{U^{\prime \prime}|\phi|^{2}}{U-c} d y+\int_{y_{1}}^{y_{2}} \frac{\left(U^{\prime \prime}\right)^{2}|\phi|^{2}}{\left|U-c_{r}\right|^{2}+c_{i}^{2}} d y .
\end{aligned}
$$

So

$$
\begin{aligned}
\int_{y_{1}}^{y_{2}}\left(\left|\phi^{\prime \prime}\right|^{2}+\alpha^{2}\left|\phi^{\prime}\right|^{2}\right) d y & =\operatorname{Re} R H S \\
& =\alpha^{2} \int_{y_{1}}^{y_{2}} \frac{U^{\prime \prime}\left(U-c_{r}\right)|\phi|^{2}}{\left|U-c_{r}\right|^{2}+c_{i}^{2}} d y+\int_{y_{1}}^{y_{2}} \frac{\left(U^{\prime \prime}\right)^{2}|\phi|^{2}}{\left|U-c_{r}\right|^{2}+c_{i}^{2}} d y \\
& =-\alpha^{2}\left(\int_{y_{1}}^{y_{2}}\left(\left|\phi^{\prime}\right|^{2}+\alpha^{2}|\phi|^{2}\right) d y\right)+\int_{y_{1}}^{y_{2}} \frac{\left(U^{\prime \prime}\right)^{2}|\phi|^{2}}{\left|U-c_{r}\right|^{2}+c_{i}^{2}} d y
\end{aligned}
$$

by (29) with $q=c_{r}$. Now (31) follows.

Then inequality (28) follows easily from (5), (30), and (31).

Remark 3.8 (stability). The inequality (27) was used in [8] to prove that there is no unstable solution to (3) when $\alpha \geq \alpha_{\max }$. Indeed, from (27), if there exists some solution $\phi$ with $\operatorname{Im} c>0$, then

$$
\begin{aligned}
-\alpha^{2} & >\frac{\int_{y_{1}}^{y_{2}}\left(\left|\phi^{\prime}\right|^{2}-K(y)|\phi|^{2}\right) d y}{\int_{y_{1}}^{y_{2}}|\phi|^{2} d y} \\
& \geq \inf _{\phi \in \mathbf{H}_{0}^{1}\left(y_{1}, y_{2}\right)} \frac{\int_{y_{1}}^{y_{2}}\left(\left|\phi^{\prime}\right|^{2}-K(y)|\phi|^{2}\right) d y}{\int_{y_{1}}^{y_{2}}|\phi|^{2} d y}=\alpha_{\max }^{2} .
\end{aligned}
$$

This proves that the condition in Theorem 1.2 is sharp for instability.

Proof of Theorem 1.7. Given $\left(c_{s}, \alpha_{s}, \phi_{s}\right)$, let $\left\{\left(c_{k}, \alpha_{k}, \phi_{k}\right)\right\}$ (with $\operatorname{Im} c_{k}>0$ ) be a sequence of solutions to the Rayleigh equation (3), as in Definition 1.6 of the introduction. Here we take $\left\|\phi_{k}\right\|_{2}=1$. By Theorem 3.3, $c_{k} \rightarrow U_{s}$. From Lemma 3.7, we get

$$
\int_{y_{1}}^{y_{2}}\left(\left|\phi_{k}^{\prime}\right|^{2}+\left|\phi_{k}^{\prime \prime}\right|^{2}\right) d y<\max \left\{\|K\|_{\infty}^{2}, 1\right\}
$$

So there is a subsequence $\left\{\phi_{n_{k}}\right\}$ of $\left\{\phi_{k}\right\}$ and $\phi_{0} \in \mathbf{H}^{2} \cap \mathbf{H}_{0}^{1}\left(y_{1}, y_{2}\right)$ such that

$$
\left\|\phi_{n_{k}}-\phi_{0}\right\|_{C^{1}} \rightarrow 0 \text { and }\left\|\phi_{0}\right\|_{2}=1 \text {. }
$$


Taking limits in

$$
\left(\frac{d^{2}}{d y^{2}}-\alpha_{n_{k}}^{2}\right) \phi_{n_{k}}-\frac{U^{\prime \prime}}{U-c_{n_{k}}} \phi_{n_{k}}=0
$$

we get

$$
-\frac{d^{2}}{d y^{2}} \phi_{0}+\frac{U^{\prime \prime}}{U-U_{s}} \phi_{0}=-\alpha_{s}^{2} \phi_{0}
$$

From the definition of $\phi_{s}$, we have $\phi_{s}=\phi_{0}$ and thus the conclusion of Theorem 1.7 follows.

TheOrem 3.9. Let $U(y)$ be in class $\mathcal{K}$. Then the set $\Xi$ of all unstable wave numbers is open. Any boundary point $\alpha$ of $\Xi$ must satisfy the condition that $-\alpha^{2}$ is a negative eigenvalue of $-\frac{d^{2}}{d y^{2}}-K(y)$ in $\mathbf{H}^{2} \cap \mathbf{H}_{0}^{1}\left(y_{1}, y_{2}\right)$.

Proof. If $\alpha \in \Xi$, then there exists $c$ with $\operatorname{Im} c>0$ such that the Rayleigh equation (3) has some solution $\phi$. Let

$$
\psi=\left(\frac{d^{2}}{d y^{2}}-\alpha^{2}\right) \phi, \quad B_{\alpha} \psi:=U \psi-U^{\prime \prime}\left(\frac{d^{2}}{d y^{2}}-\alpha^{2}\right)^{-1} \psi
$$

Then from (3) we have $B_{\alpha} \psi=c \psi$. It is easy to see that $\sigma_{e s s}\left(B_{\alpha}\right)=\left[U_{\min }, U_{\max }\right]$. So $c$ is some discrete eigenvalue of $B_{\alpha}$. Since $B_{\alpha}$ is norm continuous in $\alpha$, for any $\alpha^{\prime}$ near $\alpha$, there is also a complex $c^{\prime}$ in the spectrum of $B_{\alpha^{\prime}}$. So $\Xi$ is open. From the definition of neutral limiting modes, we know immediately that the boundary points of $\Xi$ are neutral limiting wave numbers. Then the other conclusion in the theorem follows from Theorem 1.7.

From Theorem 3.9 , we know that in order to determine $\Xi$, we only need to know the instability property near any neutral limiting wave number. This is the basis of our method in the next section for obtaining a sufficient condition for instability.

4. Proof of Theorem 1.2. Let the steady flow $U(y)$ be in the class $\mathcal{K}^{+}$. To prove Theorem 1.2, we need to study the instability near each neutral limiting wave number. Tollmien [23] heuristically showed that unstable modes exist near a neutral mode for a symmetric flow in class $\mathcal{K}^{+}$. This was later reconsidered and the asymptotic growth rate was found by C. C. Lin [17]. However, the existence of unstable modes near a neutral mode had still not been rigorously proved. Another approach was recently given in [20] for a monotone flow in class $\mathcal{K}^{+}$, where the implicit function theorem was invoked to get existence. However, because the differentiability condition was only established on half of a neighborhood, the standard implicit function theorem does not apply. Moreover, the convergence to the neutral eigenfunction in their computation was not specified. Thus, as far as we are aware, a complete proof of Tollmien's argument does not yet exist.

Therefore in this section, we rigorously prove a perturbation result of Tollmien type for flows in class $\mathcal{K}^{+}$. The existence of an unstable mode is established when the wave number is slightly to the left of a neutral wave number.

TheOrem 4.1. Suppose $U(y)$ is in class $\mathcal{K}^{+}$and $\left(\phi_{s}, \alpha_{s}, U_{s}\right)$ with $\alpha_{s}>0$ satisfies

$$
-\frac{d^{2}}{d y^{2}} \phi_{s}+\frac{U^{\prime \prime}}{U-U_{s}} \phi_{s}=-\alpha_{s}^{2} \phi_{s}
$$


with $\phi_{s}\left(y_{1}\right)=\phi_{s}\left(y_{2}\right)=0$. Then there exists $\varepsilon_{0}<0$ such that if $\varepsilon_{0}<\varepsilon<0$, there is a nontrivial solution $\phi_{\varepsilon}$ to the Rayleigh equation

$$
\left(U-U_{s}-c(\varepsilon)\right)\left(\frac{d^{2}}{d y^{2}}-\alpha(\varepsilon)^{2}\right) \phi_{\varepsilon}-U^{\prime \prime} \phi_{\varepsilon}=0
$$

with $\phi_{\varepsilon}\left(y_{1}\right)=\phi_{\varepsilon}\left(y_{2}\right)=0$. Here $\alpha(\varepsilon)=\sqrt{\varepsilon+\alpha_{s}^{2}}$ is the perturbed wave number and $U_{s}+c(\varepsilon)$ is an unstable eigenvalue with $\operatorname{Im} c(\varepsilon)>0$. Moreover, the function $c(\varepsilon)$ is differentiable in $\left(\varepsilon_{0}, 0\right)$ and

$$
\lim _{\varepsilon \rightarrow 0-} c(\varepsilon)=0
$$

$$
\lim _{\varepsilon \rightarrow 0-} c^{\prime}(\varepsilon)=\frac{\int_{y_{1}}^{y_{2}} \phi_{s}^{2}(y) d y}{\left.i \pi \sum_{k=1}^{l}\left(\left|U^{\prime}\right|^{-1} K \phi_{s}^{2}\right)\right|_{y=a_{k}}+\mathcal{P} \int_{y_{1}}^{y_{2}}\left(K(y) \phi_{s}^{2}(y)\right) /\left(U(y)-U_{s}\right) d y},
$$

where $a_{1}, \ldots, a_{l}$ are the inflection points such that $U\left(a_{k}\right)=U_{s}, k=1, \ldots, l$, and $\mathcal{P} \int_{y_{1}}^{y_{2}}$ denotes the Cauchy principal part.

Remark 4.2. As mentioned in Remark 3.2, the number of points where $U$ takes the value $U_{s}$ is finite. In formula (35), we have

$$
\left.\sum_{k=1}^{l}\left(\left|U^{\prime}\right|^{-1} K \phi_{s}^{2}\right)\right|_{y=a_{k}}>0
$$

This is due to the following two facts:

(a) The function $\phi_{s}$ must be nonzero at at least one of the points $a_{k}$. This is a corollary of Lemma 3.5 , where $c=U_{s}$ and $z_{j}=a_{j}$.

(b) We have $U^{\prime}\left(a_{k}\right) \neq 0$ for each $k$. Otherwise there exists some $k$ such that $U^{\prime}\left(a_{k}\right)=0$. Then it is easy to see that $K\left(a_{k}\right)=\infty$, which is contradictory to our assumption that $K$ is bounded.

Proof of Theorem 4.1. Define $\phi_{1}(y ; c, \varepsilon)$ and $\phi_{2}(y ; c, \varepsilon)$ to be the solutions of

$$
-\frac{d^{2}}{d y^{2}} \phi+\frac{U^{\prime \prime}}{U-U_{s}-c} \phi+\left(\alpha_{s}^{2}+\varepsilon\right) \phi=0
$$

with $\phi_{1}\left(y_{1}\right)=0, \phi_{1}^{\prime}\left(y_{1}\right)=\phi_{s}^{\prime}\left(y_{1}\right)$ and $\phi_{2}\left(y_{1}\right)=-\frac{1}{\phi_{s}^{\prime}\left(y_{1}\right)}, \phi_{2}^{\prime}\left(y_{1}\right)=0$. Here $\varepsilon<0$ and $\operatorname{Im} c>0$. Then $\phi_{1}, \phi_{2}$ are analytic in the upper half-plane as a function of $c$ and $\phi_{1}, \phi_{2}$ are independent with Wronskian 1. Now define $I(c, \varepsilon)=\phi_{1}\left(y_{2} ; c, \varepsilon\right)$. The existence of a solution to the Rayleigh equation is equivalent to the existence of a root of $I$ with $\operatorname{Im} c>0$. It will be proved by a modified Newton method, i.e., by finding a fixed point of

$$
c \rightarrow c-\frac{I(c, \varepsilon)}{\partial I /\left.\partial c\right|_{(c, \varepsilon)=(0,0)}} .
$$

Letting

$$
N(t, y ; \varepsilon, c)=\phi_{1}(t ; \varepsilon, c) \phi_{2}(y ; \varepsilon, c)-\phi_{2}(t ; \varepsilon, c) \phi_{1}(y ; \varepsilon, c),
$$


we will show that

$$
\frac{\partial I}{\partial \varepsilon}=\int_{y_{1}}^{y_{2}} N\left(y, y_{2} ; \varepsilon, c\right) \phi_{1}(y ; c, \varepsilon) d y
$$

and

$$
\frac{\partial I}{\partial c}=\int_{y_{1}}^{y_{2}} N\left(y, y_{2} ; \varepsilon, c\right) \frac{U^{\prime \prime}(y)}{\left(U(y)-U_{s}-c\right)^{2}} \phi_{1}(y ; c, \varepsilon) d y .
$$

In order to prove $(37)$ and $(38)$, notice that for $\left(c^{\prime}, \varepsilon^{\prime}\right)$ close to $(c, \varepsilon)$ with $\operatorname{Im} c^{\prime}>0$, the function $\phi_{1}\left(y ; c^{\prime}, \varepsilon^{\prime}\right)$ satisfies

$$
\begin{aligned}
-\frac{d^{2}}{d y^{2}} \phi+\frac{U^{\prime \prime}}{U-U_{s}-c} \phi & +\left(\alpha_{s}^{2}+\varepsilon\right) \phi \\
& =\left[\frac{-U^{\prime \prime}(y)\left(c^{\prime}-c\right)}{\left(U(y)-U_{s}-c\right)\left(U(y)-U_{s}-c^{\prime}\right)}-\left(\varepsilon^{\prime}-\varepsilon\right)\right] \phi .
\end{aligned}
$$

So

$$
\begin{aligned}
& \phi_{1}\left(y ; c^{\prime}, \varepsilon^{\prime}\right)=\phi_{1}(y ; c, \varepsilon) \\
& \quad-\int_{y_{1}}^{y} N(t, y ; \varepsilon, c)\left[\frac{-U^{\prime \prime}(t)\left(c^{\prime}-c\right)}{\left(U(t)-U_{s}-c\right)\left(U(t)-U_{s}-c^{\prime}\right)}-\left(\varepsilon^{\prime}-\varepsilon\right)\right] \phi_{1}\left(t ; c^{\prime}, \varepsilon^{\prime}\right) d t .
\end{aligned}
$$

Thus, letting $y=y_{2}$,

$$
\begin{aligned}
& I\left(c^{\prime}, \varepsilon^{\prime}\right)=I(c, \varepsilon) \\
& \quad+\int_{y_{1}}^{y_{2}} N\left(t, y_{2} ; \varepsilon, c\right)\left[\frac{U^{\prime \prime}(t)\left(c^{\prime}-c\right)}{\left(U(t)-U_{s}-c\right)\left(U(t)-U_{s}-c^{\prime}\right)}+\left(\varepsilon^{\prime}-\varepsilon\right)\right] \phi_{1}\left(t ; c^{\prime}, \varepsilon^{\prime}\right) d t .
\end{aligned}
$$

Identities (37) and (38) follow from this identity by letting $\left(c^{\prime}, \varepsilon^{\prime}\right)$ tend to $(c, \varepsilon)$.

Now define the triangle

$$
\Delta_{(R, b)}=\left\{c_{r}+i c_{i}|| c_{r} \mid<R c_{i}, 0<c_{i}<b\right\}
$$

and the Cartesian product

$$
E_{\left(R, b_{1}, b_{2}\right)}=\Delta_{\left(R, b_{1}\right)} \times\left(-b_{2}, 0\right)
$$

where $b_{1}, b_{2}>0$.

We make the following claims:

(a) For fixed $R,(c, \varepsilon) \in E_{\left(R, b_{1}, b_{2}\right)}, \phi_{1}(y ; c, \varepsilon)$ uniformly converges to $\phi_{s}(y)$ in $C^{1}\left[y_{1}, y_{2}\right]$ as $c \rightarrow 0, \varepsilon \rightarrow 0-$. That is, for any $\delta>0$, there exists some $b_{0}>0$ such that

$$
\left\|\phi_{1}(y ; c, \varepsilon)-\phi_{s}(y)\right\|_{C^{1}} \leq \delta
$$

for $b_{1}, b_{2}<b_{0},(c, \varepsilon) \in E_{\left(R, b_{1}, b_{2}\right)}$.

(b) The same conclusion holds true for $\phi_{2}(y ; c, \varepsilon)$. We denote $\phi_{2}(y ; 0,0)=\phi_{z}(y)$, so that $\phi_{z}\left(y_{2}\right)=-\frac{1}{\phi_{s}^{\prime}\left(y_{2}\right)}$. Then $\phi_{2}(y ; c, \varepsilon)$ uniformly converges to $\phi_{z}(y)$ in $C^{1}\left[y_{1}, y_{2}\right]$ for $(c, \varepsilon) \in E_{\left(R, b_{1}, b_{2}\right)}, c \rightarrow 0, \varepsilon \rightarrow 0-$. 
Proof of claim (a). Indeed, if it is not true, then there exists $\delta_{0}>0$ and a sequence $\left\{\left(c_{k}, \varepsilon_{k}\right)\right\}_{k=1}^{\infty},\left(c_{k}, \varepsilon_{k}\right) \rightarrow(0,0),\left|\operatorname{Re} c_{k}\right|<R \operatorname{Im} c_{k}$ such that

$$
\left\|\phi_{1}\left(y ; c_{k}, \varepsilon_{k}\right)-\phi_{s}(y)\right\|_{C^{1}} \geq \delta_{0} .
$$

Since $\left|\operatorname{Re} c_{k}\right|<R \operatorname{Im} c_{k}$ and $\operatorname{Im} c_{k}<b_{0}$, we have

$$
\left|\frac{U^{\prime \prime}(y)}{U(y)-U_{s}-c_{k}}\right| \leq|K(y)|+|K(y)| \frac{c_{k}}{U(y)-U_{s}-c_{k}}|\leq| K(y) \mid\left(1+\sqrt{R^{2}+1}\right) .
$$

Thus

$$
\left\|\frac{U^{\prime \prime}(y)}{U(y)-U_{s}-c_{k}}\right\|_{\infty} \leq\|K\|_{\infty}\left(1+\sqrt{R^{2}+1}\right)
$$

Let $\phi_{k}=\phi_{1}\left(y ; c_{k}, \varepsilon_{k}\right)$; then we have uniform bound for $\left\|\phi_{k}\right\|_{C^{2}}$ because $\phi_{k}$ satisfies an ODE (36) with uniformly bounded coefficients and the same initial value. So by the Ascoli-Arzelà lemma, there is a subsequence $\left\{\phi_{k_{i}}\right\}$ and a function $\phi_{0} \in C^{1}\left[y_{1}, y_{2}\right]$ such that

$$
\left\|\phi_{k_{i}}-\phi_{0}\right\|_{C^{1}} \rightarrow 0
$$

as $k_{i} \rightarrow \infty$. Since $\phi_{k_{i}}$ satisfies Rayleigh's equation, $\phi_{0}$ satisfies

$$
-\frac{d^{2}}{d y^{2}} \phi_{0}+\frac{U^{\prime \prime}}{U-U_{s}} \phi_{0}=-\alpha_{s}^{2} \phi_{0}
$$

with $\phi_{0}\left(y_{1}\right)=0, \phi_{0}^{\prime}\left(y_{1}\right)=\phi_{s}^{\prime}\left(y_{1}\right)$; thus $\phi_{0}=\phi_{s}$. So $\left\|\phi_{k_{i}}-\phi_{s}\right\|_{C^{1}} \rightarrow 0$, which is a contradiction to our assumption. Claim (b) follows similarly.

In the appendix we prove that

$$
\frac{\partial I}{\partial \varepsilon} \rightarrow-\frac{1}{\phi_{s}^{\prime}\left(y_{2}\right)} \int_{y_{1}}^{y_{2}} \phi_{s}^{2}(y) d y
$$

and

$$
\frac{\partial I}{\partial c} \rightarrow \frac{1}{\phi_{s}^{\prime}\left(y_{2}\right)}\left(\left.i \pi \sum_{k=1}^{l}\left(\left|U^{\prime}\right|^{-1} K \phi_{s}^{2}\right)\right|_{y=a_{k}}+\mathcal{P} \int_{y_{1}}^{y_{2}}\left(K(y) \phi_{s}^{2}(y)\right) /\left(U(y)-U_{s}\right) d y\right)
$$

uniformly in $E_{\left(R, b_{1}, b_{2}\right)}$ as $c \rightarrow 0, \varepsilon \rightarrow 0-$. Denote these limits by

$$
\begin{aligned}
& B=-\frac{1}{\phi_{s}^{\prime}\left(y_{2}\right)} \int_{y_{1}}^{y_{2}} \phi_{s}^{2}(y) d y, \\
& C=\frac{1}{\phi_{s}^{\prime}\left(y_{2}\right)} \mathcal{P} \int_{y_{1}}^{y_{2}}\left(K(y) \phi_{s}^{2}(y)\right) /\left(U(y)-U_{s}\right) d y, \\
& D=\left.\frac{\pi}{\phi_{s}^{\prime}\left(y_{2}\right)} \sum_{k=1}^{l}\left(\left|U^{\prime}\right|^{-1} K \phi_{s}^{2}\right)\right|_{y=a_{k}},
\end{aligned}
$$

where $a_{k}(k=1, \ldots, l)$ are the inflection points. Denote

$$
f(c, \varepsilon)=I(c, \varepsilon)-B \varepsilon-(C+D i) c
$$


and

$$
h(c, \varepsilon)=-\frac{B}{C+i D} \varepsilon-\frac{f(c, \varepsilon)}{C+i D} .
$$

Then by the uniform convergence of (40) and (41), for any $\delta_{0}>0$, there exists $b_{0}$ so that when $b_{1}, b_{2}<b_{0}$ we have

$$
\left|\frac{\partial f}{\partial c}\right|,\left|\frac{\partial f}{\partial \varepsilon}\right|<\delta_{0} \quad \forall(c, \varepsilon) \in E_{\left(R, b_{1}, b_{2}\right)} .
$$

So for any $(c, \varepsilon),\left(c^{\prime}, \varepsilon^{\prime}\right)$ in the convex set $E_{\left(R, b_{1}, b_{2}\right)}$,

$$
\left|f(c, \varepsilon)-f\left(c^{\prime}, \varepsilon^{\prime}\right)\right| \leq \delta_{0}\left(\left|\varepsilon-\varepsilon^{\prime}\right|+\left|c-c^{\prime}\right|\right) .
$$

Now in (43) we let $\left(c^{\prime}, \varepsilon^{\prime}\right) \rightarrow(0,0)$ and notice that

$$
\lim _{\left(c^{\prime}, \varepsilon^{\prime}\right) \rightarrow(0,0)} f\left(c^{\prime}, \varepsilon^{\prime}\right)=\lim _{\left(c^{\prime}, \varepsilon^{\prime}\right) \rightarrow(0,0)} I\left(c^{\prime}, \varepsilon^{\prime}\right)=\lim _{\left(c^{\prime}, \varepsilon^{\prime}\right) \rightarrow(0,0)} \phi_{1}\left(y_{2} ; c^{\prime}, \varepsilon^{\prime}\right)=\phi_{s}\left(y_{2}\right)=0,
$$

so we obtain

$$
|f(c, \varepsilon)| \leq \delta_{0}(|\varepsilon|+|c|) \forall(c, \varepsilon) \in E_{\left(R, b_{1}, b_{2}\right)} .
$$

Note that for fixed $\varepsilon$, a zero of $I(c, \varepsilon)$ is a fixed point of $c \rightarrow h(c, \varepsilon)$. Let $R=4\left|\frac{C}{D}\right|$ if $C \neq 0$ and $R=1$ if $C=0$. Notice that by Remark $4.2, B D<0$. Denote

$$
Q=\sqrt{R^{2}+1} \frac{-2 D B}{C^{2}+D^{2}}+1
$$

Let

$$
\delta_{0}=\frac{1}{2} \min \left\{\frac{|B C|}{Q\left(C^{2}+D^{2}\right)}, \frac{-B D}{Q\left(C^{2}+D^{2}\right)}, 1\right\} \sqrt{C^{2}+D^{2}}
$$

if $C \neq 0$ and

$$
\delta_{0}=\frac{1}{2} \min \left\{\frac{-B D}{Q\left(C^{2}+D^{2}\right)}, 1\right\} \sqrt{C^{2}+D^{2}}
$$

if $C=0$. There exists $b_{0}$ such that if $b_{1}, b_{2}<b_{0}$, then (44) and (42) hold. We choose

$$
b_{2}=\min \left\{\frac{C^{2}+D^{2}}{-2 D B \sqrt{R^{2}+1}}, 1\right\} b_{0}, \quad b_{1}=Q b_{2} .
$$

Fix $\varepsilon \in\left(-b_{2}, 0\right)$ and let

$$
b(\varepsilon)=\frac{-2 D B}{C^{2}+D^{2}} \varepsilon
$$

We will prove that

$$
h(\cdot, \varepsilon): \Delta_{(R, b(\varepsilon))} \rightarrow \Delta_{(R, b(\varepsilon))} \text { is a contraction map },
$$

with contraction ratio no greater than $\frac{1}{2}$ for all $-b_{2}<\varepsilon<0$. 
Assuming (45), the theorem follows easily. Indeed, for each $\varepsilon \in\left(-b_{2}, 0\right)$ there exists a unique $c(\varepsilon) \in \Delta_{(R, b(\varepsilon))}$ so that $h(c(\varepsilon), \varepsilon)=c(\varepsilon)$. Since for fixed $\varepsilon, h(c, \varepsilon)$ is analytic in $\Delta_{\left(R, b_{1}\right)}$ and uniformly contracting, we know that $c(\varepsilon)$ is the unique fixed point in $\Delta_{\left(R, b_{1}\right)}$ and is differentiable with respect to $\varepsilon$ in the interval $\left(-b_{2}, 0\right)$ (see $[5$, p. 25]). We now let $\varepsilon_{0}=-b_{2}$. Since $c(\varepsilon) \in \Delta_{(R, b(\varepsilon))}$, we have

$$
\lim _{\varepsilon \rightarrow 0-} c(\varepsilon)=0 .
$$

From $I(c(\varepsilon), \varepsilon)=0$, we obtain

$$
c^{\prime}(\varepsilon)=-\frac{\partial I / \partial \varepsilon}{\partial I / \partial c}
$$

So by (40) and (41), we have

$$
\lim _{\varepsilon \rightarrow 0-} c^{\prime}(\varepsilon)=\frac{\int_{y_{1}}^{y_{2}} \phi_{s}^{2}(y) d y}{\left.i \pi \sum_{k=1}^{l}\left(\left|U^{\prime}\right|^{-1} K \phi_{s}^{2}\right)\right|_{y=a_{k}}+\mathcal{P} \int_{y_{1}}^{y_{2}}\left(K(y) \phi_{s}^{2}(y)\right) /\left(U(y)-U_{s}\right) d y} .
$$

This proves (35), and the proof of Theorem 4.1 is complete assuming (45).

Now we prove (45). By our choices of $\delta_{0}, b_{0}, b_{1}, b_{2}$, and (42), we know

$$
\left|\frac{\partial h}{\partial c}\right|=\frac{1}{\sqrt{C^{2}+D^{2}}}\left|\frac{\partial f}{\partial c}\right| \leq \frac{1}{\sqrt{C^{2}+D^{2}}} \delta_{0} \leq \frac{1}{2} \forall(c, \varepsilon) \in E_{\left(R, b_{1}, b_{2}\right)} .
$$

Thus $h$ is uniformly contracting with ratio no greater than $\frac{1}{2}$ for each fixed $\varepsilon \in\left(-b_{2}, 0\right)$. We still need to show that $h(c, \varepsilon)$ maps $\Delta_{(R, b(\varepsilon))}$ to itself. If $C \neq 0$, by $(44)$ and the definitions of $b(\varepsilon), Q$, and $\delta_{0}$, we have

$$
\begin{aligned}
\left|\frac{f(c, \varepsilon)}{C+i D}\right| \leq \delta_{0} \frac{|c|+|\varepsilon|}{\sqrt{C^{2}+D^{2}}} & \leq \frac{\delta_{0}}{\sqrt{C^{2}+D^{2}}}\left(1+\sqrt{R^{2}+1} \frac{-2 D B}{C^{2}+D^{2}}\right)|\varepsilon|=\frac{\delta_{0}|\varepsilon| Q}{\sqrt{C^{2}+D^{2}}} \\
& \leq \frac{1}{2} \min \left\{\frac{|B C|}{C^{2}+D^{2}}, \frac{-B D}{C^{2}+D^{2}}\right\}|\varepsilon| .
\end{aligned}
$$

Substituting (46) into

$$
\operatorname{Re} h=\frac{-B C}{C^{2}+D^{2}} \varepsilon-\operatorname{Re} \frac{f(c, \varepsilon)}{C+i D},
$$

we readily get

$$
\frac{1}{2} \frac{|B C|}{C^{2}+D^{2}}|\varepsilon| \leq|\operatorname{Re} h| \leq 2 \frac{|B C|}{C^{2}+D^{2}}|\varepsilon| .
$$

In the same way we get

$$
\frac{1}{2} \frac{-B D}{C^{2}+D^{2}}|\varepsilon| \leq \operatorname{Im} h \leq 2 \frac{-B D}{C^{2}+D^{2}}|\varepsilon|=b(\varepsilon) .
$$

Combining (47) and (48), we have

$$
|\operatorname{Re} h| \leq 4\left|\frac{C}{D}\right| \operatorname{Im} h=R \operatorname{Im} h .
$$


So $h \in \Delta_{(R, b(\varepsilon))}$. The proof for the case $C=0$ is the same. This proves (45), and thus the proof of Theorem 4.1 is complete.

Proof of Theorem 1.2. Let $-\alpha_{m}^{2}<-\alpha_{m-1}^{2}<\cdots<-\alpha_{1}^{2}<0$ be all the negative eigenvalues of $-\frac{d^{2}}{d y^{2}}+K(y)$. Here $\alpha_{m}=\alpha_{\max }$ as defined by (7). Combining Theorems 3.9 and 4.1 , we deduce that if $\alpha \in\left(0, \alpha_{m}\right)$ and $\alpha \neq \alpha_{i}(i=1, \ldots, m)$, then there exists an unstable mode.

Now we investigate the possibility of an instability at $\alpha=\alpha_{i}(i=1, \ldots, m)$. For each $\alpha \in\left(\alpha_{i}, \alpha_{i+1}\right)$, we know that there exists some unstable eigenvalue $c(\alpha)=$ $c_{r}(\alpha)+i c_{i}(\alpha)$ with $c_{i}>0$. We claim that

$$
\text { as } \alpha \rightarrow \alpha_{i}+, c_{i}(a) \text { has some lower bound } \delta>0 \text {. }
$$

Assuming (49), we now show the existence of an unstable eigenvalue at $\alpha_{i}$. We take a sequence $\left\{\left(c_{k}, \alpha_{k}, \phi_{k}\right)\right\}_{k=1}^{\infty}$ with $\alpha_{k} \rightarrow \alpha_{i}+$ and $\operatorname{Im} c_{k} \geq \delta>0$. The function $\phi_{k}$ with $\left\|\phi_{k}\right\|_{2}=1$ satisfies the Rayleigh equation

$$
-\frac{d^{2}}{d y^{2}} \phi_{k}+\frac{U^{\prime \prime}}{U-c_{k}} \phi_{k}=-\alpha_{k}^{2} \phi_{k}
$$

By Lemma 3.7, there is an a priori bound for $\left\|\phi_{k}\right\|_{\mathbf{H}^{2}}$, so there exists some nonzero function $\phi_{0} \in \mathbf{H}^{2}$ such that $\phi_{k} \rightarrow \phi_{0}$ strongly in $\mathbf{H}^{1}$. Note that $c_{k}$ is bounded by (4). Suppose $c_{k} \rightarrow c_{0}$ with $\operatorname{Im} c_{0} \geq \delta$. Now

$$
\left\|\frac{U^{\prime \prime}}{U-c_{k}}\right\|_{\infty} \leq \frac{\left\|U^{\prime \prime}\right\|_{\infty}}{\delta}
$$

so we can pass to the limit in (50) to deduce that $\phi_{0}$ is a weak solution to

$$
-\frac{d^{2}}{d y^{2}} \phi_{0}+\frac{U^{\prime \prime}}{U-c_{0}} \phi_{0}=-\alpha_{i}^{2} \phi_{0}
$$

Since $\operatorname{Im} c_{0}>0, \frac{U^{\prime \prime}}{U-c_{0}}$ is a smooth function. So by elliptic regularity theory, $\phi_{0}$ is a classical solution. Thus at $\alpha=\alpha_{i}$, we get an unstable eigenvalue $c_{0}$.

Proof of (49). If it is not true, then there exists a sequence $\left\{\left(c_{k}, \alpha_{k}, \phi_{k}\right)\right\}_{k=1}^{\infty}$ of solutions to Rayleigh's equation, with $\alpha_{k} \rightarrow \alpha_{i}+$ and $\operatorname{Re} c_{k} \rightarrow c_{s}, \operatorname{Im} c_{k} \rightarrow 0+$. By Theorem 1.7, $c_{s}$ must equal $U_{s}$. From the proof of Theorem 1.7, we know that $\phi_{k} \rightarrow \phi_{s}$ in $C^{1}\left[y_{1}, y_{2}\right]$, where $\phi_{s}$ is a solution to

$$
-\frac{d^{2}}{d y^{2}} \phi_{s}+\frac{U^{\prime \prime}}{U-U_{s}} \phi_{s}=-\alpha_{i}^{2} \phi_{s}
$$

Multiplying (51) by $\phi_{k}$ and subtracting $\phi_{s}$ times (50), then integrating from $y_{1}$ to $y_{2}$, we get

$$
\left(\alpha_{k}^{2}-\alpha_{i}^{2}\right) \int_{y_{1}}^{y_{2}} \phi_{s} \phi_{k} d y=-\left(c_{k}-U_{s}\right) \int_{y_{1}}^{y_{2}} \frac{U^{\prime \prime} \phi_{s} \phi_{k}}{\left(U-c_{k}\right)\left(U-U_{s}\right)} d y .
$$

Let

$$
A_{k}=\int_{y_{1}}^{y_{2}} \phi_{s} \phi_{k} d y, B_{k}=-\int_{y_{1}}^{y_{2}} \frac{U^{\prime \prime} \phi_{s} \phi_{k}}{\left(U-c_{k}\right)\left(U-U_{s}\right)} d y
$$


Then

$$
\lim _{k \rightarrow \infty} A_{k}=\int_{y_{1}}^{y_{2}}\left|\phi_{s}\right|^{2} d y .
$$

In the appendix we will prove

$$
\lim _{k \rightarrow \infty} B_{k}=\mathcal{P} \int_{y_{1}}^{y_{2}} \frac{K(y) \phi_{s}^{2}}{\left(U-U_{s}\right)} d y+\left.i \pi \sum_{k=1}^{l}\left(\left|U^{\prime}\right|^{-1} K \phi_{s}^{2}\right)\right|_{y=a_{k}} .
$$

Now we have

$$
\lim _{k \rightarrow \infty} \frac{A_{k}}{B_{k}}=\frac{\int_{y_{1}}^{y_{2}}\left|\phi_{s}\right|^{2} d y}{\mathcal{P} \int_{y_{1}}^{y_{2}} \frac{K(y) \phi_{s}^{2}}{\left(U-U_{s}\right)} d y+\left.i \pi \sum_{k=1}^{l}\left(\left|U^{\prime}\right|^{-1} K \phi_{s}^{2}\right)\right|_{y=a_{k}}}=a+i b
$$

with $b<0$. Thus if $k$ is large enough,

$$
\operatorname{Im} c_{k}=\left(\alpha_{k}^{2}-\alpha_{i}^{2}\right) \operatorname{Im} \frac{A_{k}}{B_{k}}<0,
$$

which is a contradiction. So (49) is proved and the proof of Theorem 1.2 is complete.

We also have the following result about the instability at $\alpha=0$.

LEMma 4.3. If $U\left(y_{1}\right) \neq U\left(y_{2}\right)$, then at $\alpha=0$ there is also some unstable solution to the Rayleigh equation.

Proof. Let $\left\{\left(c_{k}, \alpha_{k}, \phi_{k}\right)\right\}_{k=1}^{\infty}$ be a sequence of unstable solutions with $\alpha_{k} \rightarrow 0+$. It suffices to prove that there is some positive lower bound for $\left\{\operatorname{Im} c_{k}\right\}$. Indeed the existence of an unstable solution at $\alpha=0$ would follow by the same argument as in the proof of Theorem 1.2.

Assume there is no lower bound. Then $\operatorname{Im} c_{k} \rightarrow 0, \operatorname{Re} c_{k} \rightarrow c$. Then $\phi_{k}$ converges to a neutral solution $\phi_{0} \in \mathbf{H}^{2} \cap \mathbf{H}_{0}^{1}$ satisfying equation

$$
(U-c) \phi_{0}^{\prime \prime}-U^{\prime \prime} \phi_{0}=0
$$

sectionally in each $\left(z_{i}, z_{i+1}\right)$. Here we use the same notation as immediately before Lemma 3.5. We now show that $\phi_{0}$ cannot vanish at any zero $z_{1}, \ldots, z_{k_{c}}$ of $U-c$. Indeed, if it is not true, we suppose $\phi_{0}\left(z_{i}\right)=0$ and consider (54) in $\left(z_{i}, z_{i+1}\right)$. Then

$$
\left((U-c) \phi_{0}^{\prime}-U^{\prime} \phi_{0}\right)(y) \equiv\left((U-c) \phi_{0}^{\prime}-U^{\prime} \phi_{0}\right)\left(z_{i}\right)=0
$$

for all $y$ in the interval $\left(z_{i}, z_{i+1}\right)$. So $\phi_{0}$ and $U-c$ are linearly dependent in $\left(z_{i}, z_{i+1}\right)$. Thus $\phi_{0}\left(z_{i+1}\right)=0$. Repeating the process, we know that $\phi_{0}\left(z_{i}\right)=0$ for all $i=$ $1, \ldots, k_{c}$ and there is some constant $b$ such that $U(y)-c=b \phi_{0}(y)$ for all $y$ in $\left(y_{1}, y_{2}\right)$. This implies that $U\left(y_{1}\right)=U\left(y_{2}\right)=0$, which is a contradiction.

Thus $\phi_{0}$ takes a nonzero value at each zero of $U-c$ and $\phi_{0}$ is the limit of unstable eigenfunctions. By the proof of Theorem 3.3 we know that $c$ must equal $U_{s}$. Now by the argument in the last part of the proof of Theorem 1.2, we know that there is no perturbation of the neutral mode at $\alpha=0$ to its right neighborhood. This contradiction shows that the $\operatorname{Im} c_{k}$ has some positive lower bound. The proof of the lemma is finished.

Remark 4.4. If $U\left(y_{1}\right)=U\left(y_{2}\right)$, it is possible that at $\alpha=0$ there is no unstable solution to (3). One such example is $U(y)=\cos 6 y$, whose complete spectrum was found in [12] and for which there is no growing mode at $\alpha=0$. 
5. Rotating flows. In this section, we consider the radially symmetric steady flows in an annulus $0<R_{1} \leq r \leq R_{2}$. Using polar coordinates $(r, \theta)$, we rewrite the vorticity equation (1) as

$$
\partial_{t} \Delta \psi+\frac{1}{r} \frac{\partial \psi}{\partial \theta} \frac{\partial}{\partial r} \Delta \psi-\frac{\partial \psi}{\partial r} \frac{1}{r} \frac{\partial}{\partial \theta} \Delta \psi=0
$$

Here $\psi$ is the stream function and

$$
\left(u_{r}, u_{\theta}\right)=\left(\frac{1}{r} \frac{\partial \psi}{\partial \theta},-\frac{\partial \psi}{\partial r}\right), \quad \omega=-\Delta \psi=\frac{1}{r} \frac{\partial}{\partial r}\left(r u_{\theta}\right)-\frac{1}{r} \frac{\partial u_{r}}{\partial \theta}
$$

are the velocity and vorticity, respectively. And $\psi$ is constant on $r=R_{j}(j=1,2)$. The steady flow is $\left(u_{r}, u_{\theta}\right)=(0, r \Omega)$, with $\Omega=\Omega(r)$ the steady angular velocity. The linearized equation about this steady flow is

$$
\partial_{t} \Delta \tilde{\psi}+\Omega \frac{\partial}{\partial \theta} \Delta \tilde{\psi}+\left(\frac{\partial}{\partial r} Z\right) \frac{1}{r} \frac{\partial \tilde{\psi}}{\partial \theta}=0
$$

with $\tilde{\psi}$ constant on $r=R_{j}(j=1,2)$ and the steady vorticity $Z=2 \Omega+r \frac{d \Omega}{d r}$. Taking $\tilde{\psi}(r, \theta, t)=\phi(r) \exp (s t+i n \theta)$ and letting $D_{*}=\frac{d}{d r}+\frac{1}{r}, D=\frac{d}{d r}$ (following the notation in $[6])$, we rewrite $(55)$ as

$$
(s+i n \Omega)\left(D_{*} D-n^{2} / r^{2}\right) \phi-i n r^{-1}\left(r D^{2} \Omega+3 D \Omega\right) \phi=0,
$$

with $\phi\left(R_{1}\right)=\phi\left(R_{2}\right)=0$ and $n$ a positive integer. Letting $c=\frac{s}{i n}$, we get the rotating Rayleigh equation (8). Instability would mean that there exists a solution to (8) with $\operatorname{Im} c>0$. In this section we study the flows such that the function $K(r)$ defined by (9) is positive and bounded, which we still denote by class $\mathcal{K}^{+}$.

We are interested only in the case when $\alpha$ is a positive integer of the following extended Rayleigh equation:

$$
(\Omega-c)\left(D_{*} D-\alpha^{2} / r^{2}\right) \phi-r^{-1}\left(r D^{2} \Omega+3 D \Omega\right) \phi=0,
$$

with $\phi\left(R_{1}\right)=\phi\left(R_{2}\right)=0$. However, by embedding the original problem into a family of problems (57) depending on a continuous positive parameter $\alpha$, we can use the same idea as in the shear flow case. For that purpose, first we need to prove the rotating versions of some results used in the shear flow case. We give detailed proofs only when they are really different. First is the extension of Lemma 3.5 to the rotating case. Let $r_{1}<r_{2}<\cdots<r_{k_{c}}$ be the zeros of $\Omega(y)-c$ and let $S_{0}$ be the complement of the set of points $\left\{r_{i}\right\}$ in the interval $\left[R_{1}, R_{2}\right]$. Here $c$ is any real number in the range of $\Omega(y)$. Let $r_{0}=R_{1}$ and $r_{k_{c}+1}=R_{2}$. Note that for the rotating flows in class $\mathcal{K}^{+}, k_{c}$ is finite for any $c$ by the same argument as in Remark 3.2.

LEMma 5.1. Let $\phi$ satisfy (57) on $S_{0}$ with real $\alpha>1$ and real $c$ in the range of $\Omega$. We assume $\phi$ is sectionally continuous on the open intervals $\left(r_{j}, r_{j+1}\right), j=$ $0,1, \ldots, k_{c}$. Then $\phi$ cannot vanish at both endpoints of any of the intervals $\left(r_{j}, r_{j+1}\right)$ unless it vanishes identically in that interval.

Proof. The function $\phi$ satisfies

$$
(\Omega-c)\left(D_{*} D-\alpha^{2} / r^{2}\right) \phi-r^{-1}\left(r D^{2} \Omega+3 D \Omega\right) \phi=0,
$$

which is the same as

$$
(\Omega-c) D^{2} \phi-\phi D^{2} \Omega+(\Omega-c) r^{-1} D \phi-3 r^{-1} \phi D \Omega=(\Omega-c) \frac{\alpha^{2}}{r^{2}} \phi .
$$


We multiply both sides of the above by $r^{2}$ to get

$$
(\Omega-c) r^{2} D^{2} \phi-r^{2} \phi D^{2} \Omega+(\Omega-c) r D \phi-3 r \phi D \Omega=(\Omega-c) \alpha^{2} \phi .
$$

We have

$$
\begin{aligned}
\text { LHS } & =(\Omega-c)\left[D^{2}\left(r^{2} \phi\right)-4 r D \phi-2 \phi\right]-r^{2} \phi D^{2} \Omega+(\Omega-c) r D \phi-3 r \phi D \Omega \\
& =(\Omega-c) D^{2}\left(r^{2} \phi\right)-r^{2} \phi D^{2} \Omega-3(D(r \phi)(\Omega-c)+r \phi D \Omega)+\phi(\Omega-c) \\
& =D\left((\Omega-c) D\left(r^{2} \phi\right)-r^{2} \phi D \Omega\right)-3 D((r \phi)(\Omega-c))+\phi(\Omega-c) .
\end{aligned}
$$

So (58) becomes (using ' to replace $D$ )

$$
\left((\Omega-c)\left(r^{2} \phi\right)^{\prime}-r^{2} \phi \Omega^{\prime}\right)^{\prime}-3((r \phi)(\Omega-c))^{\prime}=\left(\alpha^{2}-1\right) \phi(\Omega-c) .
$$

Suppose $\phi$ vanishes at $r_{i}$ and $r_{i+1}$. Here we mean $\phi\left(r_{i}+\right)$ for $\phi\left(r_{i}\right)$ and $\phi\left(r_{i+1}-\right)$ for $\phi\left(r_{i+1}\right)$ when studying (59) in $\left[r_{i}, r_{i+1}\right]$. From the definition of $r_{i}$ we know that $\Omega-c$ has constant sign in $\left(r_{i}, r_{i+1}\right)$. If $r_{i} \neq R_{1}(i \neq 0)$, then $\Omega\left(r_{i}\right)-c=0$. Let $\tilde{r} \leq r_{i+1}$ be the nearest zero of $\phi$ in $\left(r_{i}, r_{i+1}\right]$. Since (59) is a real equation, we may assume $\phi$ is real and nonnegative on the interval $\left(r_{i}, \tilde{r}\right)$ and that $\left(r^{2} \phi\right)^{\prime}\left(r_{i}\right) \geq 0$ and $\left(r^{2} \phi\right)^{\prime}(\tilde{r}) \leq 0$. Integrating $(59)$ over $\left(r_{i}, \tilde{r}\right)$, we get

$$
(\Omega(\tilde{r})-c)\left(r^{2} \phi\right)^{\prime}(\tilde{r})=\left(\alpha^{2}-1\right) \int_{r_{i}}^{\tilde{r}} \phi(\Omega-c) d r^{\prime},
$$

since $\phi$ vanishes at the endpoints $r_{i}, \tilde{r}$.

If $\tilde{r}=r_{i+1}$, then the left-hand side above must be zero. Hence $\phi$ is identically zero on $\left(r_{i}, r_{i+1}\right)$. On the other hand, if $\tilde{r}<r_{i+1}$, then $\Omega(\tilde{r}) \neq c$ and

$$
\left(r^{2} \phi\right)^{\prime}(\tilde{r})=\left(\alpha^{2}-1\right) \int_{r_{i}}^{\tilde{r}} \frac{\left(\Omega\left(r^{\prime}\right)-c\right)}{(\Omega(\tilde{r})-c)} \phi\left(r^{\prime}\right) d r^{\prime},
$$

which could not hold true unless $\phi=0$ on $\left[r_{i}, \tilde{r}\right]$. But the second order ODE (58) is regular on $\left(r_{i}, r_{i+1}\right)$; thus $\tilde{r}$ could not be a cluster point of nontrivial $\phi$. Thus $\phi$ must be identically zero on $\left(r_{i}, r_{i+1}\right)$.

If $i=0$, then we repeat the same argument with the right endpoint of the interval $\left(R_{1}, r_{1}\right)$.

We need Howard's semicircle theorem for the rotating case, which seems not to have been proven in the literature. So we give a proof here.

Lemma 5.2. If $\alpha>1$, then for the extended Rayleigh equation (57) to have a solution, $c$ (with $\operatorname{Im} c>0)$ must lie in the semicircle

$$
\left(c_{r}-\frac{1}{2}\left(\Omega_{\min }+\Omega_{\max }\right)\right)^{2}+c_{i}^{2} \leq\left(\frac{1}{2}\left(\Omega_{\min }-\Omega_{\max }\right)\right)^{2},
$$

where $\Omega_{\min }$ and $\Omega_{\max }$ are the minimum and maximum of $\Omega(r)$ in $\left[R_{1}, R_{2}\right]$.

Proof. Let $\phi$ be a solution to (57). As in the proof of the last lemma, $\phi$ satisfies (59), which we can rewritten as

$$
\left(r\left(\phi^{\prime}(\Omega-c) r-((\Omega-c) r)^{\prime} \phi\right)\right)^{\prime}=\left(\alpha^{2}-1\right) \phi(\Omega-c) .
$$


The above identity is equivalent to

$$
\left(r^{3}(\Omega-c)^{2}\left(\frac{\phi}{(\Omega-c) r}\right)^{\prime}\right)^{\prime}=\left(\alpha^{2}-1\right) \phi(\Omega-c) .
$$

Now $\psi=\frac{\phi}{(\Omega-c) r}$ is a regular function since $\operatorname{Im} c \neq 0$. Then (62) becomes

$$
\left(r^{3}(\Omega-c)^{2} \psi\right)^{\prime}=\left(\alpha^{2}-1\right) r(\Omega-c)^{2} \psi
$$

Multiplying (63) by $\psi^{*}$ (conjugate of $\psi$ ) and integrating it, we obtain

$$
\int_{R_{1}}^{R_{2}}(\Omega-c)^{2}\left(r^{3}\left|\psi^{\prime}\right|^{2}+\left(\alpha^{2}-1\right) r|\psi|^{2}\right) d r=0 .
$$

The rest of the proof is the same as in the case of shear flows [14], [6]. We repeat it here for completeness. Let

$$
P \equiv r^{3}\left|\psi^{\prime}\right|^{2}+\left(\alpha^{2}-1\right) r|\psi|^{2}
$$

Then (64) becomes

$$
\int_{R_{1}}^{R_{2}}(\Omega-c)^{2} P d r=0
$$

The function $P$ is nonnegative and not identically zero. Comparing the real and imaginary parts of $(65)$, we get

$$
\int_{R_{1}}^{R_{2}}\left(\left(\Omega-c_{r}\right)^{2}-c_{i}^{2}\right) P d r=0 \quad \text { and } \quad 2 c_{i} \int_{R_{1}}^{R_{2}}\left(\Omega-c_{r}\right) P d r=0 .
$$

Observe that

$$
\begin{aligned}
0 & \geq \int_{R_{1}}^{R_{2}}\left(\Omega-\Omega_{\min }\right)\left(\Omega-\Omega_{\max }\right) P d r \\
& =\int_{R_{1}}^{R_{2}}\left\{\left(c_{r}^{2}+c_{i}^{2}\right)-\left(\Omega_{\min }+\Omega_{\max }\right) c_{r}+\Omega_{\min } \Omega_{\max }\right\} P d r
\end{aligned}
$$

where $(66)$ is used. So

$$
\left(c_{r}^{2}+c_{i}^{2}\right)-\left(\Omega_{\min }+\Omega_{\max }\right) c_{r}+\Omega_{\min } \Omega_{\max } \leq 0
$$

and the conclusion follows.

We also have an a priori bound for unstable solutions. The proof of the following lemma is essentially the same as that of Lemma 3.7 in the shear flow case. So we state only the result.

Lemma 5.3. Denote $\omega(r)=r D^{2} \Omega+3 D \Omega$. For any solution $(\alpha, c, \phi)$ to (57) with $\alpha$ real positive, $c=c_{r}+i c_{i}\left(c_{i}>0\right)$, and $\|\phi\|_{2}=1$, we have the identities

$$
\int_{R_{1}}^{R_{2}} r|D \phi|^{2} d r+\alpha^{2} \int_{R_{1}}^{R_{2}} \frac{1}{r}|\phi|^{2} d r+\int_{R_{1}}^{R_{2}} \frac{\omega(r)|\phi|^{2}(\Omega-q)}{|\Omega-c|^{2}} d r=0 \forall q \in \mathbf{R},
$$




$$
\int_{R_{1}}^{R_{2}}\left(\left|D_{*} D \phi\right|^{2} r^{3}+2 \alpha^{2} r|D \phi|^{2} d r+\alpha^{4} \frac{1}{r}|\phi|^{2}\right) d r=\int_{R_{1}}^{R_{2}} r \frac{\omega(r)^{2}}{|\Omega-c|^{2}}|\phi|^{2} d r .
$$

For a flow of class $\mathcal{K}^{+}$, we have the inequalities

$$
\int_{R_{1}}^{R_{2}}\left(r(D \phi)^{2}+\alpha^{2} \frac{1}{r}|\phi|^{2}\right) d r<\int_{R_{1}}^{R_{2}} K(r) \phi^{2} d r
$$

and

$$
\int_{R_{1}}^{R_{2}}\left(\left|D_{*} D \phi\right|^{2} r^{3}+2 \alpha^{2} r|D \phi|^{2} d r+\alpha^{4} \frac{1}{r}|\phi|^{2}\right) d r<R_{2}\|\omega\|_{\infty} \int_{R_{1}}^{R_{2}} K(r) \phi^{2} d r .
$$

In particular, we have the a priori estimate $\|\phi\|_{\mathbf{H}^{2}} \leq C(\Omega)$, where $C(\Omega)$ is some constant depending only on $\Omega$.

Indeed, (67), (68), (69), (70) are the analogues of (29), (31), (27), and (28), respectively. Their proofs are similar to that of the shear flow case.

Remark 5.4. From (69) we see that a necessary condition for instability in the rotating case is

$$
\inf _{\phi \in \mathbf{H}_{0}^{1}\left(R_{1}, R_{2}\right)} \frac{\int_{R_{1}}^{R_{2}} r(D \phi)^{2} d r-\int_{R_{1}}^{R_{2}} K(r) \phi^{2} d r}{\int_{R_{1}}^{R_{2}} \frac{1}{r} \phi^{2} d r}<-1,
$$

since $\alpha$ must be a positive integer.

We now define the neutral limiting modes for the rotating case.

DeFinition 5.5. The triple $\left(c_{s}, \alpha_{s}, \phi_{s}\right)$ with $c_{s}$ real and $\alpha_{s}>1$ is said to be a neutral limiting mode if it is the limit of growing solutions $\left(c_{k}, \alpha_{k}, \phi_{k}\right)$ (with $\operatorname{Im} c_{k}>0$ ) of the extended Rayleigh equation (57). The precise notion of convergence of $\phi_{k}$ to $\phi_{s}$ is made clear in Lemma 5.6. Formally $\left(c_{s}, \alpha_{s}, \phi_{s}\right)$ ought to satisfy

$$
\left(\Omega-c_{s}\right)\left(D_{*} D-\alpha_{s}^{2} / r^{2}\right) \phi_{s}-r^{-1} \omega(r) \phi_{s}=0 .
$$

We call $c_{s}$ the neutral limiting phase speed and $\alpha_{s}$ the neutral limiting wave number.

The following is the analogue of Lemma 3.6.

Lemma 5.6. Let $\left\{\left(c_{k}, \alpha_{k}, \phi_{k}\right)\right.$ (with $\left.\left.\operatorname{Im} c_{k}>0\right)\right\}_{k=1}^{\infty}$ be the solutions to the extended Rayleigh equation (57) with $\left\|\phi_{k}\right\|=1$, and let $\left(c_{k}, \alpha_{k}\right)$ converge to $\left(c_{s}, \alpha_{s}\right)$ with $\alpha_{s}>1$. Then $\phi_{k}$ converges uniformly to a function $\phi_{s}$ on any compact subset of $S_{0}, \phi_{s}^{\prime \prime}$ exists on $S_{0}$, and $\phi_{s}$ satisfies (71).

We state the following results about neutral limiting modes without proof. They are the analogues of Theorems 1.7 and 3.9, respectively.

LEMMA 5.7. If the rotating flow is in class $\mathcal{K}^{+}$, then for any neutral limiting mode $\left(c_{s}, \alpha_{s}, \phi_{s}\right)$ with $\alpha_{s}>1$, we must have $c_{s}=\Omega_{s}$, and $\phi_{s} \in \mathbf{H}^{2} \cap \mathbf{H}_{0}^{1}\left(R_{1}, R_{2}\right)$ must satisfy

$$
\left(D_{*} D-\alpha_{s}^{2} / r^{2}\right) \phi_{s}+r^{-1} K(r) \phi_{s}=0 .
$$

Lemma 5.8. Let $\Omega(y)$ be as in Theorem 1.3. Let $\Xi$ be the set of all unstable wave numbers greater than 1 . Then $\Xi$ is open and any real boundary point $\alpha_{s}$ of $\Xi$ is either 1 or some wave number satisfying (72) for some nontrivial $\phi_{s}$ in $\mathbf{H}^{2} \cap \mathbf{H}_{0}^{1}\left(R_{1}, R_{2}\right)$.

We also have a perturbation result near neutral modes, the analogue of Theorem 4.1. 
Theorem 5.9. Suppose $\Omega(y)$ is in class $\mathcal{K}^{+}$and $\left(\phi_{s}, \alpha_{s}, \Omega_{s}\right)\left(\alpha_{s}>1\right)$ satisfies

$$
\left(\Omega-\Omega_{s}\right)\left(D_{*} D-\alpha_{s}^{2} / r^{2}\right) \phi_{s}-r^{-1} \omega(r) \phi_{s}=0,
$$

with $\phi_{s}\left(R_{1}\right)=\phi_{s}\left(R_{2}\right)=0$. Then there exists $\varepsilon_{0}<0$ such that if $\varepsilon_{0}<\varepsilon<0$, there is a nontrivial solution $\phi_{\varepsilon}$ to the extended Rayleigh equation

$$
\left(\Omega-\Omega_{s}-c(\varepsilon)\right)\left(D_{*} D-\alpha(\varepsilon)^{2} / r^{2}\right) \phi_{\varepsilon}-r^{-1} \omega(r) \phi_{\varepsilon}=0,
$$

with $\phi_{\varepsilon}\left(R_{1}\right)=\phi_{\varepsilon}\left(R_{2}\right)=0$. Here $\alpha(\varepsilon)=\sqrt{\varepsilon+\alpha_{s}^{2}}$ is the perturbed wave number and $\Omega_{s}+c(\varepsilon)$ is an unstable eigenvalue with $\operatorname{Im} c(\varepsilon)>0$. The function $c(\varepsilon)$ is differentiable in $\left(-\varepsilon_{0}, 0\right)$ and

$$
\lim _{\varepsilon \rightarrow 0-} c(\varepsilon)=0
$$

$$
\lim _{\varepsilon \rightarrow 0-} c^{\prime}(\varepsilon)=\frac{\int_{R_{1}}^{R_{2}} \frac{1}{r} \phi_{s}^{2}(r) d r}{\left.i \pi \sum_{k=1}^{l}\left(\left|\Omega^{\prime}\right|^{-1} K \phi_{s}^{2}\right)\right|_{y=r_{k}}+P \int_{R_{1}}^{R_{2}}\left(K(r) \phi_{s}^{2}(r)\right) /\left(\Omega(r)-\Omega_{s}\right) d r},
$$

where $r_{1}, \ldots, r_{l}$ are the points such that $\Omega(r)=\Omega_{s}$ and $\mathcal{P} \int_{y_{1}}^{y_{2}}$ denotes the Cauchy principal part.

Proof of Theorem 1.3. If (11) is satisfied, we know that for any $\alpha \in\left(1, \alpha_{\max }\right)$, there is an unstable solution to the extended Rayleigh equation (57). The proof is essentially the same as that of Theorem 1.2, by using Theorem 5.9 and Lemma 5.8, so we skip it here. If condition (12) is satisfied, then $\alpha_{\max }>2$, and we get instability at $n=2$ for the rotating Rayleigh equation (8).

Now we turn to the case when $1<\alpha_{\max } \leq 2$ and $\Omega\left(R_{1}\right) \neq \Omega\left(R_{2}\right)$. We want to show that there exists an unstable mode for $n=1$. This is the bottom case for rotating flows. Now for each $\alpha \in\left(1, \alpha_{\max }\right)$, we already have an unstable mode. We shall show that the growth rate $\operatorname{Im} c(\alpha)$ has some positive lower bound when $\alpha$ tends to 1 . Assuming this, we can find some unstable mode for $\alpha=1$ by using the same argument as in the proof of Theorem 1.2.

We now prove that $\operatorname{Im} c(\alpha)$ has some positive lower bound. The argument we use here is similar to that in the proof of Lemma 4.3. Supposing otherwise, we can find a sequence $\left\{\left(c_{k}, \alpha_{k}, \phi_{k}\right)\right\}_{k=1}^{\infty}$ with $\alpha_{k} \rightarrow 1+, \operatorname{Im} c_{k} \rightarrow 0, \operatorname{Re} c_{k} \rightarrow c$. The convergence of $\left\{c_{k}\right\}$ is guaranteed by (60), from which we also know that $c$ is in the range of $\Omega$. Because of Lemma 5.3, $\left\|\phi_{k}\right\|_{\mathbf{H}^{2}}$ is uniformly bounded. So there exists some nonzero $\phi_{0}$ in $\mathbf{H}^{2} \cap \mathbf{H}_{0}^{1}$ such that a subsequence $\left\{\phi_{n_{k}}\right\}$ converges to it in the $\mathbf{C}^{1}$ sense. By passing to the limit in the equation

$$
\left(\Omega-c_{n_{k}}\right)\left(D_{*} D-\alpha_{n_{k}}^{2} / r^{2}\right) \phi_{n_{k}}-r^{-1}\left(r D^{2} \Omega+3 D \Omega\right) \phi_{n_{k}}=0,
$$

we deduce that the function $\phi_{0}$ satisfies

$$
(\Omega-c)\left(D_{*} D-1 / r^{2}\right) \phi_{0}-r^{-1}\left(r D^{2} \Omega+3 D \Omega\right) \phi_{0}=0
$$

sectionally in each $\left(r_{i}, r_{i+1}\right)$. Here $r_{1}<r_{2}<\cdots<r_{k_{c}}$ are the zeros of $\Omega(y)-c$ and $r_{0}=R_{1}, r_{k_{c}+1}=R_{2}$. By (59), $\phi_{0}$ satisfies

$$
\left((\Omega-c)\left(r^{2} \phi_{0}\right)^{\prime}-r^{2} \phi_{0} \Omega^{\prime}\right)^{\prime}-3\left(\left(r \phi_{0}\right)(\Omega-c)\right)^{\prime}=0
$$


which is equivalent to

$$
\left(r\left(((\Omega-c) r)^{\prime} \phi_{0}-\phi_{0}^{\prime}(\Omega-c) r\right)\right)^{\prime}=0 .
$$

From (76), we deduce that $\phi_{0}$ must be nonzero at each point $r_{i}\left(i=1, \ldots, k_{c}\right)$. Indeed, supposing otherwise, by the same argument as in the proof of Lemma 4.3, we can show that there is some constant $b$ such that $(\Omega(r)-c) r=b \phi_{0}(r)$ in $\left[R_{1}, R_{2}\right]$. This implies that $\Omega\left(R_{1}\right)=\Omega\left(R_{2}\right)$, a contradiction. Now by the same argument as in the last part of the proof of Theorem 1.2, we can show that there is no perturbation of the neutral mode at $\alpha=1$ to its right neighborhood. This contradiction shows that $\operatorname{Im} c(\alpha)$ is bounded below and $\operatorname{Im} c>0$. Thus $\phi_{0}$ satisfying (75) is an unstable solution to the rotating Rayleigh equation (8).

Combining this result with Remark 5.4, we deduce that the condition (11) is sharp for instability when $\Omega\left(R_{1}\right) \neq \Omega\left(R_{2}\right)$. This finishes the proof of Theorem 1.3.

6. Unbounded flows. We now consider the unbounded shear flows. We prove Theorem 1.5(i) only for the flow $U(y)$ defined on $(-\infty,+\infty)$. The proof of Theorem 1.5(i) for the shear flows defined on the half line is similar. The flow with $U(-\infty)=$ $U(+\infty)$ is called a jet and the one with $U(-\infty) \neq U(+\infty)$ is called a shear layer, as in $[7]$.

Proof of Theorem 1.5(i). We divide the proof into several steps.

Step 1. First we observe that for any real $c, U(y)=c$ holds for only a finite number of points. Otherwise, there exists some real $c_{0}$ and an infinite sequence $\left\{y_{n}\right\}$ such that $U\left(y_{n}\right)=c_{0}$ for each $n$. Then $\left\{y_{n}\right\}$ must be bounded by our condition that $U( \pm \infty)$ are obtained at only a finite number of points. So there exists some $y_{0}$ such that a subsequence $\left\{y_{n_{k}}\right\}$ converges to it. Since $U(y)$ is a $\mathbf{C}^{2}$ function, we deduce that $U\left(y_{0}\right)=c_{0}$ and $U^{\prime}\left(y_{0}\right)=U^{\prime \prime}\left(y_{0}\right)=0$. So $y_{0}$ is an inflection point and $c_{0}$ equals the inflection value $U_{s}$. But then $K(y)$ defined by (5) is unbounded at $y_{0}$ since $U^{\prime}\left(y_{0}\right)=0$. This is a contradiction.

Since $K(y) \rightarrow 0$ as $y \rightarrow \infty$, it is easy to see that $-\frac{d^{2}}{d y^{2}}-K(y)$ is a relatively compact perturbation of $-\frac{d^{2}}{d y^{2}}$ defined on $\mathbf{H}^{2}(\mathbf{R})$. So by Weyl's theorem [21]

$$
\sigma_{\mathrm{ess}}\left(-\frac{d^{2}}{d y^{2}}-K(y)\right)=\sigma_{\mathrm{ess}}\left(-\frac{d^{2}}{d y^{2}}\right)=(0,+\infty)
$$

Thus $-\frac{d^{2}}{d y^{2}}-K(y)$ has only a discrete set of negative eigenvalues which can accumulate only at 0 . Let $-\alpha_{0}^{2}<-\alpha_{1}^{2}<\cdots<-\alpha_{k}^{2}<\cdots<0$ denote all the negative eigenvalues of the operator $-\frac{d^{2}}{d y^{2}}-K(y)$ on $\mathbf{H}^{2}(\mathbf{R})$. We fix some $\alpha$ in $\left(0, \alpha_{0}\right)$ and assume $\alpha \neq \alpha_{i}$ for each $i \geq 1$. Let $I_{n}=(-n, n)$ and let $L_{n}$ denote the operator $-\frac{d^{2}}{d y^{2}}-K(y)$ on $\mathbf{H}^{2}\left(I_{n}\right) \cap \mathbf{H}_{0}^{1}\left(I_{n}\right)$. Take $n$ large enough so that all the inflection points of $K(y)$ are included in $I_{n}$. Denote by $-\alpha_{0, n}^{2}$ the lowest eigenvalue of $L_{n}$. Then by the result of [3], $-\alpha_{0, n}^{2}$ converges to $-\alpha_{0}^{2}$ as $n$ tends to infinity. So by taking $n$ large enough, $\alpha$ is in $\left(0, \alpha_{0, n}\right)$. Now since $\left.U(y)\right|_{I_{n}}$ is clearly in class $\mathcal{K}^{+}$, by applying Theorem 1.2 to this truncated flow, we obtain a solution $\phi_{n}$ in $\mathbf{H}^{2}\left(I_{n}\right) \cap \mathbf{H}_{0}^{1}\left(I_{n}\right)$ satisfying the Rayleigh equation with $c_{n}\left(\operatorname{Im} c_{n}>0\right)$, that is,

$$
\left(U-c_{n}\right)\left(\frac{d^{2}}{d y^{2}}-\alpha^{2}\right) \phi_{n}-U^{\prime \prime} \phi_{n}=0
$$

in $I_{n}$. 
Step 2. Now we have a sequence $\left\{\left(\phi_{n}, c_{n}\right)\right\}$ satisfying (77) with $\left\|\phi_{n}\right\|_{\mathbf{L}^{2}\left(I_{n}\right)}=1$. By Lemma 3.7, we have $\left\|\phi_{n}\right\|_{\mathbf{H}^{2}\left(I_{n}\right)} \leq C$ (where $C$ is some constant depending only on $\left.\|K\|_{\infty}\right)$. We extend $\phi_{n}$ to a function in $\mathbf{H}^{2}(\mathbf{R})$ by setting it to be zero on $I_{n}^{c}$. For convenience, we still use $\phi_{n}$ to denote the extended function. Then $\left\|\phi_{n}\right\|_{\mathbf{H}^{2}(\mathbf{R})} \leq C$. So $\phi_{n}$ converges weakly in $\mathbf{H}^{2}(\mathbf{R})$ to a function $\phi_{0} \in \mathbf{H}^{2}(\mathbf{R})$. We shall show that $\phi_{0}$ is not identically zero.

Let $n_{0}$ be sufficiently large such that

$$
K(y)<\frac{1}{2} \alpha^{2} \quad \text { if } y \in I_{n_{0}}^{c} .
$$

From (27) we have

$$
\int_{I_{n}}\left(\left|\phi_{n}^{\prime}\right|^{2}+\alpha^{2}\left|\phi_{n}\right|^{2}\right) d y<\int_{I_{n}} K(y)\left|\phi_{n}\right|^{2} d y
$$

For any $n>n_{0}$, from (78) and $K>0$ we have

$$
\begin{aligned}
\int_{I_{n_{0}}} K(y)\left|\phi_{n}\right|^{2} d y & >\alpha^{2}-\int_{I_{n_{0}}^{c} \cap I_{n}} K(y)\left|\phi_{n}\right|^{2} d y \\
& >\alpha^{2}-\frac{1}{2} \alpha^{2} \int_{I_{n_{0}}^{c} \cap I_{n}}\left|\phi_{n}\right|^{2} d y \\
& >\frac{1}{2} \alpha^{2} .
\end{aligned}
$$

Since $\phi_{n}$ converges strongly in $\mathbf{H}^{1}\left(I_{n_{0}}\right)$ to $\phi_{0}$, from (79) we get

$$
\int_{I_{n_{0}}} K(y)\left|\phi_{0}\right|^{2} d y \geq \frac{1}{2} \alpha^{2} .
$$

This shows that $\phi_{0}$ is nontrivial.

Step 3. By Howard's semicircle theorem (see (4)), $\left\{c_{n}\right\}$ is bounded. Supposing $c_{n}$ to converge to $c_{0}$, we shall show that $\operatorname{Im} c_{0}>0$. Otherwise, $c_{0}$ is some real number in the range of $U(y)$. From the a priori $\mathbf{H}^{2}$ bound provided by Lemma 3.7, we deduce that $\phi_{n}$ converges to $\phi_{0}$ locally in $\mathbf{C}^{1}$. Suppose $z_{1}<\cdots<z_{k_{0}}$ are all the points such that $U\left(z_{i}\right)=c_{0}$. Then by taking limit $k \rightarrow \infty$ in (77), we deduce that $\phi_{0}$ satisfies

$$
\left(U-c_{0}\right)\left(\frac{d^{2}}{d y^{2}}-\alpha^{2}\right) \phi_{0}-U^{\prime \prime} \phi_{0}=0
$$

within each interval $\left(-\infty, z_{1}\right),\left(z_{1}, z_{2}\right), \ldots,\left(z_{k_{0}}, \infty\right)$. Since $\phi_{0} \neq 0$, by the proof of Lemma 3.5, we deduce that $\phi_{0}\left(z_{i}\right) \neq 0$ for some $z_{i}$. Then by the same argument as in the proof of Theorem 3.3, we know that $c_{0}$ must be an inflection value of $U(y)$, which can only be $U_{s}$. Now

$$
\frac{U^{\prime \prime}}{U-c_{0}}=-K(y)
$$

is a bounded continuous function and $\phi_{0}$ is in $\mathbf{C}^{1}$. So from (80), $\phi_{0}$ satisfies

$$
\left(\frac{d^{2}}{d y^{2}}-\alpha^{2}\right) \phi_{0}+K(y) \phi_{0}=0
$$


on $\mathbf{R}$ and $\phi_{0} \in \mathbf{H}^{2}(\mathbf{R})$. Thus $-\alpha^{2}$ is a negative eigenvalue of

$$
-\frac{d^{2}}{d y^{2}}-K(y)
$$

on $\mathbf{H}^{2}(\mathbf{R})$, which is a contradiction to our assumption that $\alpha \neq \alpha_{i}$ for all $i$. So we must have $\operatorname{Im} c_{0}>0$.

Now $\frac{U^{\prime \prime}}{U-c_{0}}$ is a bounded continuous function. By passing to limits in (77), we deduce that $\phi_{0} \neq 0$ satisfies the Rayleigh equation (3) on the whole line, with $\operatorname{Im} c_{0}>$ 0 . We thus get instability for each wave number $\alpha$ in $\left(0, \alpha_{0}\right)$ such that $\alpha \neq \alpha_{i}(i \geq 1)$.

Step 4. Now we investigate the possibility of an instability at $\alpha=\alpha_{i}(i \geq 1)$. The argument is the same as in the proof of Theorem 1.2 for the case when a wave number equals some neutral limiting wave number. We only sketch it here. For each $\alpha>\alpha_{i}$, we get instability by the previous steps. The main point is still to show that the growth rate $\operatorname{Im} c(\alpha)$ has some positive lower bound when $\alpha \rightarrow \alpha_{i}+$. Supposing otherwise, we get a sequence $\left\{\left(c_{k}, \alpha_{k}, \phi_{k}\right)\right\}_{k=1}^{\infty}$ of solutions to Rayleigh's equation (3), with $\alpha_{k} \rightarrow \alpha_{i}+$ and $\operatorname{Re} c_{k} \rightarrow c_{s}, \operatorname{Im} c_{k} \rightarrow 0+$. It is not difficult to see that we can extend Theorem 1.7 to the current case, so $c_{s}$ must equal $U_{s}$. By the same argument as in Step 2, we deduce that $\phi_{k}$ converges to some $\phi_{s} \neq 0$ weakly in $\mathbf{H}^{2}(\mathbf{R})$ and locally in $\mathbf{C}^{1}$. Now $\phi_{s}$ satisfies

$$
-\frac{d^{2}}{d y^{2}} \phi_{s}+\frac{U^{\prime \prime}}{U-U_{s}} \phi_{s}=-\alpha_{i}^{2} \phi_{s}
$$

By Remark 7.2 in the appendix, the analogues of the two limits (52) and (53) still hold true. That is, we have

$$
\int_{\mathbf{R}} \phi_{k} \phi_{s} \rightarrow \int_{\mathbf{R}}\left|\phi_{s}\right|^{2} d y
$$

and

$$
\int_{\mathbf{R}} \frac{K(y) \phi_{s} \phi_{k}}{U-c_{k}} d y \rightarrow \mathcal{P} \int_{\mathbf{R}} \frac{K(y) \phi_{s}^{2}}{\left(U-U_{s}\right)} d y+\left.i \pi \sum_{i=1}^{l}\left(\left|U^{\prime}\right|^{-1} K \phi_{s}^{2}\right)\right|_{y=a_{i}} .
$$

Here $a_{1}, \ldots, a_{l}$ are all the inflection points and $c_{k} \rightarrow U_{s}$ with $\operatorname{Im} c_{k}>0$. Then the rest of the proof just follows what we did in the last part of the proof of Theorem 1.2. So we skip it here.

Proof of Theorem 1.5(ii). Assume $\phi$ is odd on the whole line. Fix $\alpha$ in the interval $\left(\alpha_{2 k_{0}-1}, \alpha_{2 k_{0}-2}\right)$ for some $k_{0}$. Using the same notation as in Step 1 of the proof of (i), we get the truncated operator $L_{n}$ defined on $I_{n}=(-n, n)$. Let $-\alpha_{0, n}^{2}<-\alpha_{1, n}^{2}<$ $\cdots<-\alpha_{k, n}^{2}<\cdots<0$ be all the negative eigenvalues of $L_{n}$. By the result in [3], $L_{n}$ has at least $2 k_{0}$ negative eigenvalues when $n$ is large enough and $\left(-\alpha_{0, n}^{2}, \ldots,-\alpha_{2 k_{0}-1, n}^{2}\right)$ converges to $\left(-\alpha_{0}^{2}, \ldots,-\alpha_{2 k_{0}-1}^{2}\right)$ as $n$ tends to infinity. So for $n$ large enough, $\alpha$ is in the interval $\left(\alpha_{2 k_{0}-1, n}, \alpha_{2 k_{0}-2, n}\right)$. Since $\left.U(y)\right|_{I_{n}}$ is odd, by Theorem 1.4 we obtain an unstable solution $\phi_{n}$ satisfying (77) in $I_{n}$, with $c_{n}=i \lambda_{n}\left(\lambda_{n}>0\right)$. We get the same a priori bound as in Lemma 3.7 directly as follows. By Lemma 3.5 with $q=0$, we have

$$
\int_{I_{n}}\left(\left|\phi_{n}^{\prime}\right|^{2}+\alpha^{2}\left|\phi_{n}\right|^{2}+\frac{U^{\prime \prime} U}{\left|U-i \lambda_{n}\right|^{2}}\left|\phi_{n}\right|^{2}\right) d y=0 .
$$


Thus

$$
\begin{aligned}
\int_{I_{n}}\left(\left|\phi_{n}^{\prime}\right|^{2}+\alpha^{2}\left|\phi_{n}\right|^{2}\right) d y & =\int_{I_{n}} K(y) \frac{U^{2}}{U^{2}+\lambda_{n}^{2}}\left|\phi_{n}\right|^{2} d y \\
& \leq \int_{I_{n}}|K(y)|\left|\phi_{n}\right|^{2} d y .
\end{aligned}
$$

Similarly, we get from (31) that

$$
\int_{I_{n}}\left(\left|\phi_{n}^{\prime \prime}\right|^{2}+2 \alpha^{2}\left|\phi_{n}^{\prime}\right|^{2}+\alpha^{4}|\phi|^{2}\right) d y<\int_{I_{n}} K(y)^{2}\left|\phi_{n}\right|^{2} d y .
$$

From (84) and (85), we have $\left\|\phi_{n}\right\|_{\mathbf{H}^{2}(\mathbf{R})} \leq C\left(\|K\|_{\infty}\right)$. So $\phi_{n}$ converges to some $\phi_{0}$ weakly in $\mathbf{H}^{2}(\mathbf{R})$. Let $\lambda_{n}$ converge to some nonnegative $\lambda_{0}$. By the same argument as in Steps 2 and 3 of the proof of (i), we show that $\phi_{0} \neq 0$ and $\lambda_{0}>0$. Then we get an unstable solution $\phi_{0}$ to the Rayleigh equation on the whole line, with the unstable eigenvalue $c=i \lambda_{0}$. The proof of Theorem 1.5(ii) is thus finished.

Using the same argument as in the proof of Lemma 4.3, we can show that if $U(-\infty) \neq U(+\infty)$ (the shear layer case), then at $\alpha=0$ there is also an unstable solution to the Rayleigh equation. This coincides with the conclusion in [7], which was deduced from the asymptotic expansion in the long wave limit.

7. Appendix. In this appendix, we prove some asymptotic formulas used in the proof of Theorems 4.1 and 1.2.

Lemma 7.1. Assume a sequence of differentiable functions $\left\{\psi_{k}\right\}_{k=1}^{\infty}$ converges in $C^{1}\left[y_{1}, y_{2}\right]$ to $\psi_{0}(y)$. Let $c_{k}=p_{k}+i b_{k}\left(b_{k}>0\right)$ converge to 0 . Denote $W_{k}(y)=$ $U(y)-U_{s}-p_{k}$. Then we have following limits:

$$
\begin{gathered}
\lim _{k \rightarrow \infty} \int_{y_{1}}^{y_{2}} \frac{\psi_{k}(y) W_{k}(y)}{W_{k}(y)^{2}+b_{k}^{2}} d y=\mathcal{P} \int_{y_{1}}^{y_{2}} \frac{\psi_{0}(y)}{U(y)-U_{s}} d y, \\
\lim _{k \rightarrow \infty} \int_{y_{1}}^{y_{2}} \frac{\psi_{k}(y) W_{k}(y)^{3}}{\left(W_{k}(y)^{2}+b_{k}^{2}\right)^{2}} d y=\mathcal{P} \int_{y_{1}}^{y_{2}} \frac{\psi_{0}(y)}{U(y)-U_{s}} d y, \\
\lim _{k \rightarrow \infty} \int_{y_{1}}^{y_{2}} \frac{\psi_{k}(y) b_{k}}{W_{k}(y)^{2}+b_{k}^{2}} d y=\left.\pi \sum_{i=1}^{l}\left(\left|U^{\prime}\right|^{-1} \psi_{0}\right)\right|_{y=a_{i}}, \\
\lim _{k \rightarrow \infty} \int_{y_{1}}^{y_{2}} \frac{\psi_{k}(y) b_{k}^{3}}{\left(W_{k}(y)^{2}+b_{k}^{2}\right)^{2}} d y=\left.\frac{1}{2} \pi \sum_{i=1}^{l}\left(\left|U^{\prime}\right|^{-1} \psi_{0}\right)\right|_{y=a_{i}}, \\
\lim _{k \rightarrow \infty} \int_{y_{1}}^{y_{2}} \frac{\psi_{k}(y) b_{k}^{2} W_{k}}{\left(W_{k}(y)^{2}+b_{k}^{2}\right)^{2}} d y=\lim _{k \rightarrow \infty} \int_{y_{1}}^{y_{2}} \frac{\psi_{k}(y) b_{k}^{3}}{\left(W_{k}(y)^{2}+b_{k}^{2}\right)^{2}} d y=0 .
\end{gathered}
$$

Here $a_{1}, \ldots, a_{l}$ are all the points such that $U(y)=U_{s}$, and we assume $U^{\prime}(y) \neq 0$ at each $a_{k}$. 
Proof. Let $\left\|\psi_{k}\right\|_{\mathbf{C}^{1}} \leq M$ (independent of $k$ ). For $\delta_{0}>0$, we can find $r_{0}>0$ such that $\left|U^{\prime}(y)\right| \geq \delta_{0}$ for all $y$ in the set $E_{r_{0}}=\cup_{i=1}^{l} I\left(a_{i} ; r_{0}\right)$. Here $I\left(a_{i} ; r_{0}\right)=$ $\left(a_{i}-r_{0}, a_{i}+r_{0}\right)$. Taking $k$ large enough, then we know there are exactly $l$ points such that $W_{k}(y)=0$, one in each $I\left(a_{i} ; r_{0}\right)$, which we denote by $a_{i}^{(k)}$.

Proof of (86). By the definition of Cauchy principal part, for any $\varepsilon>0$, there exists some $r_{1}>0$ such that if $0<r<r_{1}$, then

$$
\left|\int_{E_{r}^{c}} \frac{\psi_{0}(y)}{U(y)-U_{s}} d y-\mathcal{P} \int_{y_{1}}^{y_{2}} \frac{\psi_{0}(y)}{U(y)-U_{s}} d y\right|<\frac{\varepsilon}{3} .
$$

Now

$$
\frac{\psi_{k}(y) W_{k}(y)}{W_{k}(y)^{2}+b_{k}^{2}} \rightarrow \frac{\psi_{0}(y)}{U(y)-U_{s}}
$$

in $E_{r}^{c}$ uniformly as $k \rightarrow \infty$. So if $k$ is large enough, then

$$
\left|\int_{E_{r}^{c}} \frac{\psi_{k}(y) b_{k}}{W_{k}(y)^{2}+b_{k}^{2}} d y-\int_{E_{r}^{c}} \frac{\psi_{0}(y)}{U(y)-U_{s}} d y\right|<\frac{\varepsilon}{3} .
$$

Let $r<\min \left\{r_{0}, r_{1}\right\}$. We estimate the integral on each $I\left(a_{i} ; r\right)$. Suppose $U$ is increasing on $I\left(a_{1} ; r\right)$. Let

$$
\begin{gathered}
t=W_{k}(y), \quad t_{1}^{k}=W_{k}\left(a_{1}+r\right), \quad t_{0}^{k}=W_{k}\left(a_{1}-r\right), \\
\tilde{\psi}_{k}(t)=\psi_{k}\left(W_{k}(t)\right) \frac{1}{U^{\prime}\left(W_{k}(t)\right)} .
\end{gathered}
$$

Then

$$
\begin{aligned}
& \int_{I\left(a_{1} ; r\right)} \frac{\psi_{k}(y) W_{k}(y)}{W_{k}(y)^{2}+b_{k}^{2}} d y \\
= & \int_{t_{0}^{k}}^{t_{1}^{k}} \frac{\tilde{\psi}_{k}(t) t}{t^{2}+b_{k}^{2}} d t=\tilde{\psi}_{k}(0) \int_{t_{0}^{k}}^{t_{1}^{k}} \frac{t}{t^{2}+b_{k}^{2}} d t+\left(\int_{t_{0}^{k}}^{t_{1}^{k}} \frac{\left(\tilde{\psi}_{k}(t)-\tilde{\psi}_{k}(0)\right) t}{t^{2}+b_{k}^{2}} d t\right)=I+I I .
\end{aligned}
$$

We have

$$
\left|t_{1}^{k}-t_{0}^{k}\right| \leq\left\|U^{\prime}\right\|_{\infty} 2 r
$$

and $\left|\tilde{\psi}_{k}^{\prime}(t)\right| \leq M^{\prime}$ (independent of $k$ ) in $I\left(a_{1} ; r\right)$. So

$$
\|I I\| \leq M^{\prime}\left|t_{1}^{k}-t_{0}^{k}\right| \leq M^{\prime}\left\|U^{\prime}\right\|_{\infty} 2 r
$$

And

$$
I=\tilde{\psi}_{k}(0) \frac{1}{2} \ln \frac{t_{1}^{k}+b_{k}^{2}}{t_{0}^{k}+b_{k}^{2}}
$$

tends to zero as $k$ tends to infinity for any fixed $r>0$. Thus by choosing $r$ small enough and then letting $k$ large, we have

$$
\left|\int_{E_{r}} \frac{\psi_{k}(y) W_{k}(y)}{W_{k}(y)^{2}+b_{k}^{2}} d y\right|<\frac{\varepsilon}{3} .
$$


Combining with (91) and (92), we have for $k$ large enough

$$
\left|\int_{y_{1}}^{y_{2}} \frac{\psi_{k}(y) W_{k}(y)}{W_{k}(y)^{2}+b_{k}^{2}} d y-\mathcal{P} \int_{y_{1}}^{y_{2}} \frac{\psi_{0}(y)}{U(y)-U_{s}} d y\right|<\varepsilon
$$

This ends the proof of (86).

We prove (87) and (90) in the same way.

Proof of (88). For any fixed $r$,

$$
\lim _{k \rightarrow \infty} \int_{E_{r}^{c}} \frac{\psi_{k}(y) b_{k}}{W_{k}(y)^{2}+b_{k}^{2}} d y=0 .
$$

So we only need to consider the integral on each small interval $I\left(a_{i} ; r\right)$. Using the same notation as above, we have

$$
\begin{aligned}
& \int_{I\left(a_{1} ; r\right)} \frac{\psi_{k}(y) b_{k}}{W_{k}(y)^{2}+b_{k}^{2}} d y \\
& =\int_{t_{0}^{k}}^{t_{1}^{k}} \frac{\tilde{\psi}_{k}(t) b_{k}}{t^{2}+b_{k}^{2}} d t=\tilde{\psi}_{k}(0) \int_{t_{0}^{k}}^{t_{1}^{k}} \frac{b_{k}}{t^{2}+b_{k}^{2}} d t+\left(\int_{t_{0}^{k}}^{t_{1}^{k}} \frac{\left(\tilde{\psi}_{k}(t)-\tilde{\psi}_{k}(0)\right) b_{k}}{t^{2}+b_{k}^{2}} d t\right)=I+I I .
\end{aligned}
$$

Then it is easy to see

$$
|I I| \leq \frac{1}{2} M^{\prime}\left|t_{1}^{k}-t_{0}^{k}\right| \leq M^{\prime}\left\|U^{\prime}\right\|_{\infty} r
$$

Since

$$
\begin{aligned}
\lim _{k \rightarrow \infty} \tilde{\psi}_{k}(0) & =\lim _{k \rightarrow \infty} \psi_{k}\left(a_{1}^{(k)}\right) \frac{1}{U^{\prime}\left(a_{1}^{(k)}\right)}=\psi_{0}\left(a_{1}\right) \frac{1}{U^{\prime}\left(a_{1}\right)} \\
\lim _{k \rightarrow \infty} t_{1}^{k} & =U\left(a_{1}+r\right)-U\left(a_{1}\right)>0, \quad \lim _{k \rightarrow \infty} t_{0}^{k}=U\left(a_{1}-r\right)-U\left(a_{1}\right)<0
\end{aligned}
$$

so we have

$$
\lim _{k \rightarrow \infty} I=\lim _{k \rightarrow \infty} \tilde{\psi}_{k}(0) \int_{\frac{t_{0}^{k}}{b_{k}}}^{\frac{t_{0}^{k}}{b_{k}}} \frac{1}{t^{2}+1} d t=\psi_{0}\left(a_{1}\right) \frac{1}{U^{\prime}\left(a_{1}\right)} \int_{-\infty}^{+\infty} \frac{1}{t^{2}+1} d t=\pi \psi_{0}\left(a_{1}\right) \frac{1}{U^{\prime}\left(a_{1}\right)} .
$$

Summing the contributions from each $I\left(a_{i} ; r\right)$, we deduce (88). The proof of $(89)$ is the same by noticing that

$$
\int_{\mathbf{R}} \frac{1}{\left(1+t^{2}\right)^{2}} d t=\frac{1}{2} \pi
$$

Now (53) follows directly from the above lemma. Indeed letting $\psi_{k}=K(y) \phi_{s} \phi_{k}$, $c_{k}=p_{k}+i b_{k}$, we can write $B_{k}$ in (53) as

$$
B_{k}=\int_{y_{1}}^{y_{2}} \frac{\psi_{k}(y) W_{k}(y)}{W_{k}(y)^{2}+b_{k}^{2}} d y+i \int_{y_{1}}^{y_{2}} \frac{\psi_{k}(y) b_{k}}{W_{k}(y)^{2}+b_{k}^{2}} d y .
$$

Since

$$
\psi_{k} \rightarrow \psi_{0}=K(y) \phi_{s}^{2} \quad \text { in } C^{1}\left[y_{1}, y_{2}\right] \text { as } k \rightarrow \infty,
$$


by (86) and (88) we have

$$
\lim _{k \rightarrow \infty} B_{k}=\mathcal{P} \int_{y_{1}}^{y_{2}} \frac{K(y) \phi_{s}^{2}}{\left(U-U_{s}\right)} d y+\left.i \pi \sum_{k=1}^{l}\left(\left|U^{\prime}\right|^{-1} K \phi_{s}^{2}\right)\right|_{y=a_{k}}
$$

Remark 7.2. The limit (53) still holds for the case $\left[y_{1}, y_{2}\right]=(-\infty,+\infty)$ or $(0,+\infty)$ under the assumption that $\left\{\phi_{k}\right\}_{k=1}^{\infty}$ converges weakly in $\mathbf{L}^{2}\left(y_{1}, y_{2}\right)$ and locally in $C^{1}$ to $\phi_{0}(y)$. To see it, we notice that for fixed $r_{0}>0$,

$$
\lim _{k \rightarrow \infty} \int_{\mathbf{R} / E_{r_{0}}} \frac{K(y) \phi_{s} \phi_{k} W_{k}(y)}{W_{k}(y)^{2}+b_{k}^{2}} d y=\int_{\mathbf{R} / E_{r_{0}}} \frac{K(y) \phi_{s}^{2}}{U(y)-U_{s}} d y
$$

by weak convergence of $\phi_{k}$. We can deal with the integral on each small interval $I\left(a_{i} ; r_{0}\right)$ in the same way as in the proof of Lemma 7.1, noticing that the $\mathbf{C}^{1}$ norm of $\phi_{k}$ is locally uniformly bounded.

To prove (41) we need the following lemma.

Lemma 7.3. Assume a sequence of differentiable functions $\left\{\Gamma_{k}\right\}_{k=1}^{\infty}$ converges in $C^{1}\left[y_{1}, y_{2}\right]$ to $\Gamma_{0}(y)$. Let $c_{k}=p_{k}+i b_{k}$ converge to 0 , where $b_{k}>0$ and $\left|p_{k}\right| \leq R b_{k}$. Then we have

$$
\lim _{k \rightarrow \infty}-\int_{y_{1}}^{y_{2}} \frac{U^{\prime \prime}(y) \Gamma_{k}(y)}{\left(U-U_{s}-c_{k}\right)^{2}} d y=\mathcal{P} \int_{y_{1}}^{y_{2}} \frac{K(y) \Gamma_{0}(y)}{U(y)-U_{s}} d y+\left.i \pi \sum_{i=1}^{l}\left(\left|U^{\prime}\right|^{-1} K \phi_{0}\right)\right|_{y=a_{i}} .
$$

Proof. Denote $W_{k}(y)=U(y)-U_{s}-p_{k}$. We have

$$
\begin{aligned}
& \int_{y_{1}}^{y_{2}} \frac{-U^{\prime \prime}(y) \Gamma_{k}(y)}{\left(U-U_{s}-c_{k}\right)^{2}} d y \\
= & \int_{y_{1}}^{y_{2}} \frac{K(y) \Gamma_{k}(y)\left(U(y)-U_{s}\right)}{\left(W_{k}(y)-i b_{k}\right)^{2}} d y \\
= & \int_{y_{1}}^{y_{2}} \frac{K(y) \Gamma_{k}(y)\left(W_{k}+p_{k}\right)\left(W_{k}^{2}+2 i b_{k} W_{k}-b_{k}^{2}\right)}{\left(W_{k}^{2}+b_{k}^{2}\right)^{2}} d y \\
= & \int_{y_{1}}^{y_{2}} \frac{K(y) \Gamma_{k}(y) W_{k}^{3}}{\left(W_{k}^{2}+b_{k}^{2}\right)^{2}} d y+2 i \int_{y_{1}}^{y_{2}} \frac{K(y) \Gamma_{k}(y) b_{k} W_{k}^{2}}{\left(W_{k}^{2}+b_{k}^{2}\right)^{2}} d y-\int_{y_{1}}^{y_{2}} \frac{K(y) \Gamma_{k}(y) W_{k} b_{k}^{2}}{\left(W_{k}^{2}+b_{k}^{2}\right)^{2}} d y \\
& \quad+\int_{y_{1}}^{y_{2}} \frac{K(y) \Gamma_{k}(y) p_{k}\left(W_{k}^{2}-b_{k}^{2}\right)}{\left(W_{k}^{2}+b_{k}^{2}\right)^{2}} d y+2 i \int_{y_{1}}^{y_{2}} \frac{K(y) \Gamma_{k}(y) p_{k} b_{k} W_{k}}{\left(W_{k}^{2}+b_{k}^{2}\right)^{2}} d y \\
= & I+I I+I I I+I V+V .
\end{aligned}
$$

Now we estimate each term separately. By (87) in Lemma 7.1, we have

$$
\lim _{k \rightarrow \infty} I=\mathcal{P} \int_{y_{1}}^{y_{2}} \frac{K(y) \Gamma_{0}(y)}{U(y)-U_{s}} d y
$$


By (88) and (89), we have

$$
\begin{aligned}
\lim _{k \rightarrow \infty} I I & =2 i\left(\lim _{k \rightarrow \infty} \int_{y_{1}}^{y_{2}} \frac{K(y) \Gamma_{k}(y) b_{k}}{W_{k}^{2}+b_{k}^{2}} d y-\lim _{k \rightarrow \infty} \int_{y_{1}}^{y_{2}} \frac{K(y) \Gamma_{k}(y) b_{k}^{3}}{\left(W_{k}^{2}+b_{k}^{2}\right)^{2}} d y\right) \\
& =2 i\left(\left.\pi \sum_{i=1}^{l}\left(\left|U^{\prime}\right|^{-1} K \phi_{0}\right)\right|_{y=a_{i}}-\left.\frac{1}{2} \pi \sum_{i=1}^{l}\left(\left|U^{\prime}\right|^{-1} K \phi_{0}\right)\right|_{y=a_{i}}\right) \\
& =\left.i \pi \sum_{i=1}^{l}\left(\left|U^{\prime}\right|^{-1} K \phi_{0}\right)\right|_{y=a_{i}} .
\end{aligned}
$$

By (90), $\lim _{k \rightarrow \infty} I I I=0$. Notice that

$$
\begin{aligned}
I V & =\frac{p_{k}}{b_{k}} \int_{y_{1}}^{y_{2}} \frac{K(y) \Gamma_{k}(y) b_{k}\left(W_{k}^{2}+b_{k}^{2}-2 b_{k}^{2}\right)}{\left(W_{k}^{2}+b_{k}^{2}\right)^{2}} d y \\
& =\frac{p_{k}}{b_{k}}\left(\int_{y_{1}}^{y_{2}} \frac{K(y) \Gamma_{k}(y) b_{k}}{W_{k}^{2}+b_{k}^{2}} d y-2 \int_{y_{1}}^{y_{2}} \frac{K(y) \Gamma_{k}(y) b_{k}^{3}}{\left(W_{k}^{2}+b_{k}^{2}\right)^{2}} d y\right) \\
& =\frac{p_{k}}{b_{k}} V I .
\end{aligned}
$$

By (88) and (89), we have

$$
\begin{aligned}
\lim _{k \rightarrow \infty} V I & =\lim _{k \rightarrow \infty} \int_{y_{1}}^{y_{2}} \frac{K(y) \Gamma_{k}(y) b_{k}}{W_{k}^{2}+b_{k}^{2}} d y-2 \lim _{k \rightarrow \infty} \int_{y_{1}}^{y_{2}} \frac{K(y) \Gamma_{k}(y) b_{k}^{3}}{\left(W_{k}^{2}+b_{k}^{2}\right)^{2}} d y \\
& =\left.\pi \sum_{i=1}^{l}\left(\left|U^{\prime}\right|^{-1} K \Gamma_{0}\right)\right|_{y=a_{i}}-\left.2 \frac{1}{2} \pi \sum_{i=1}^{l}\left(\left|U^{\prime}\right|^{-1} K \Gamma_{0}\right)\right|_{y=a_{i}} \\
& =0 .
\end{aligned}
$$

Combining it with the fact that $\left|p_{k}\right| \leq R b_{k}$, we have $\lim _{k \rightarrow \infty} I V=0$. Now for the last term, we have

$$
V=2 i \frac{p_{k}}{b_{k}} \int \frac{K(y) \Gamma_{k}(y) b_{k}^{2} W_{k}}{\left(W_{k}^{2}+b_{k}^{2}\right)^{2}} d y=2 i \frac{p_{k}}{b_{k}} V I I .
$$

By (90), $\lim _{k \rightarrow \infty} V I I=0$. Thus we also have $\lim _{k \rightarrow \infty} V=0$ since $\left|p_{k}\right| \leq R b_{k}$.

Combining the five terms above, we get (93).

Proof of (41). We must show that (41) holds uniformly in $E_{\left(R, b_{1}, b_{2}\right)}$. Supposing otherwise, we can find some $\delta_{0}>0$ and a sequence $\left(c_{k}, \varepsilon_{k}\right)$ in $E_{R, b_{1}, b_{2}}$ with $\max \left\{b_{1}^{k}, b_{2}^{k}\right\}$ tending to 0 such that

$$
\left|\frac{\partial I}{\partial c}\left(c_{k}, \varepsilon_{k}\right)-(C+i D)\right|>\delta_{0}
$$

where

$$
C+i D=\frac{1}{\phi_{s}^{\prime}\left(y_{2}\right)}\left(\left.i \pi \sum_{k=1}^{l}\left(\left|U^{\prime}\right|^{-1} K \phi_{s}^{2}\right)\right|_{a_{k}}+\mathcal{P} \int_{y_{1}}^{y_{2}}\left(K(y) \phi_{s}^{2}(y)\right) /\left(U(y)-U_{s}\right) d y\right) .
$$


But

$$
\frac{\partial I}{\partial c}\left(c_{k}, \varepsilon_{k}\right)=\int_{y_{1}}^{y_{2}} \frac{-U^{\prime \prime}(y) \Gamma_{k}(y)}{\left(U-U_{s}-c_{k}\right)^{2}} d y
$$

where

$$
\Gamma_{k}(y)=-N\left(y, y_{2} ; \varepsilon_{k}, c_{k}\right) \phi_{1}\left(y ; c_{k}, \varepsilon_{k}\right) .
$$

Since $\Gamma_{k}$ converges in $\mathbf{C}^{1}$ to

$$
-N\left(y, y_{2} ; 0,0\right) \phi_{1}(y ; 0,0)=\frac{1}{\phi_{s}^{\prime}\left(y_{2}\right)} \phi_{s}^{2}(y)
$$

Lemma 7.3 implies

$$
\begin{aligned}
& \lim _{k \rightarrow \infty} \frac{\partial I}{\partial c}\left(c_{k}, \varepsilon_{k}\right) \\
& =\frac{1}{\phi_{s}^{\prime}\left(y_{2}\right)}\left(\left.i \pi \sum_{k=1}^{l}\left(\left|U^{\prime}\right|^{-1} K \phi_{s}^{2}\right)\right|_{a_{k}}+\mathcal{P} \int_{y_{1}}^{y_{2}}\left(K(y) \phi_{s}^{2}(y)\right) /\left(U(y)-U_{s}\right) d y\right) \\
& =C+i D,
\end{aligned}
$$

which is a contradiction. Thus the uniform convergence of (41) is proved.

Acknowledgments. The author is truly indebted to his advisor, Walter Strauss, for continuous encouragement, guidance, and many helpful discussions. He especially thanks Louis Howard, whose very helpful comments and discussions have improved this paper. The author also thanks Yan Guo and Claude Bardos for useful comments and discussions. Finally, he would like to thank the referee for his comments.

\section{REFERENCES}

[1] C. Bardos, Y. Guo, and W. Strauss, Stable and unstable ideal plane flows, Chinese Ann. Math., 23B (2002), pp. 149-164.

[2] M. B. Banerjee, R. G. Shandil, K. S. Shirkot, and D. Sharma, Importance of Tollmien's counter example, Stud. Appl. Math., 105 (2000), pp. 191-202.

[3] P. B. Bailey, W. N. Everitt, J. Weidmann, and A. Zettl, Regular approximations of singular Sturm-Liouville problems, Results Math., 23 (1993), pp. 3-22.

[4] L. Belenkaya, S. Friedlander, And V. Yudovich, The unstable spectrum of oscillating shear flows, SIAM J. Appl. Math., 59 (1999), pp. 1701-1715.

[5] S. N. Chow and J. Hale, Methods of Bifurcation Theory, Springer-Verlag, New York, 1982.

[6] P. G. Drazin and W. H. Reid, Hydrodynamic Stability, Cambridge Monogr. Mech. Appl. Math., Cambridge University Press, Cambridge, UK, 1981.

[7] P. G. Drazin and L. N. Howard, The instability to long waves of unbounded parallel inviscid flow, J. Fluid Mech., 14 (1962), pp. 257-283.

[8] P. G. Drazin and L. N. Howard, Hydrodynamical stability of parallel flow of inviscid fluid, in Advances in Applied Mechanics, Vol. 7, G. Kuerti, ed., Academic Press, New York, 1966, pp. 1-89.

[9] L. D. FADDEEv, On the theory of the instability of a stationary plane-parallel flows of an ideal fluid, Zap. Naučn. Sem. Leningrad. Otdel. Mat. Inst. Steklov. (LOMI), 21 (1971), pp. $164-172$.

[10] R. FJøRTOFT, Application of integral theorems in deriving criteria of stability of laminar flow and for baroclinic circular vortex, Geofys. Publ. Norske Vid.-Akad. Oslo, 17 (1950), pp. $1-52$.

[11] S. Friedlander, W. Strauss, and M. M. Vishik, Nonlinear instability in an ideal fluid, Ann. Inst. H. Poincaré Anal. Non Linéaire, 14 (1997), pp. 187-204. 
[12] S. Friedlander and L. N. Howard, Instability in parallel flow revisited, Stud. Appl. Math., 101 (1998), pp. 1-21.

[13] E. Grenier, On the nonlinear instability of Euler and Prandtl equations, Comm. Pure. Appl. Math., 53 (2000), pp. 1067-1091.

[14] L. N. Howard, Note on a paper of John W. Miles, J. Fluid Mech., 10 (1961), pp. 509-512.

[15] L. N. HowARD, The number of unstable modes in hydrodynamic stability problems, J. Mécanique, 3 (1964), p. 433.

[16] Lord RAyleigh, On the stability or instability of certain fluid motions, Proc. London Math. Soc., 9 (1880), pp. 57-70.

[17] C. C. Lin, The Theory of Hydrodynamic Stability, Cambridge University Press, Cambridge, UK, 1955.

[18] Z. LiN, Instability of periodic BGK waves, Math. Res. Lett., 8 (2001), pp. 521-534.

[19] Z. Lin, Some stability and instability criteria for ideal plane flows, Comm. Math. Phys., submitted.

[20] C. Marchioro and M. Pulvirenti, Mathematical Theory of Incompressible Nonviscous Flu$i d s$, Springer-Verlag, New York, 1994.

[21] M. Reed And B. Simon, Methods of Modern Mathematical Physics, Vol. 4, Academic Press, New York, 1978.

[22] D. H. Sattinger, On the Rayleigh problem in hydrodynamic stability, SIAM J. Appl. Math., 15 (1967), pp. 419-425.

[23] W. Tollmien, Ein Allgemeines Kriterium der Instabitität laminarer Geschwindigkeitsverteilungen, Nachr. Ges. Wiss. Göttingen Math. Phys., 50 (1935), pp. 79-114. 